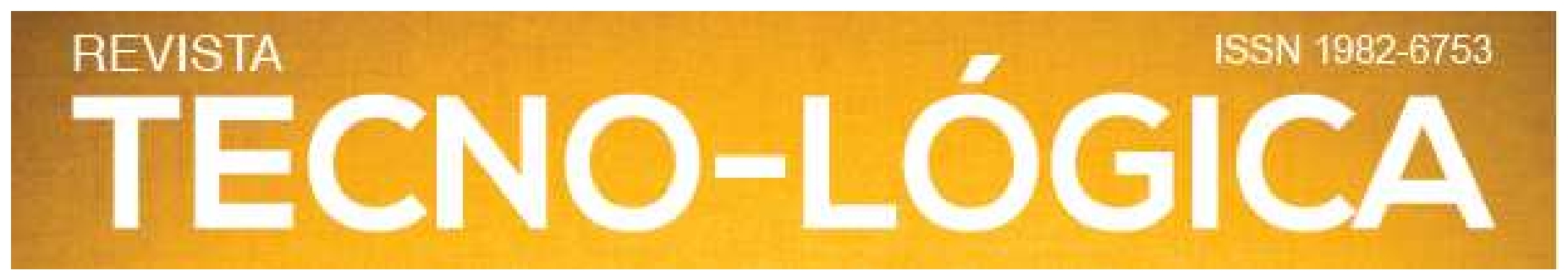

\title{
DEPENDÊNCIA E CORRELAÇÃO ESPACIAL DOS ATRIBUTOS DE SOLO EM FUNÇÃO DO ARRANJO AMOSTRAL
}

Evandro Gelain ${ }^{1}$, Eduardo Leonel Bottega ${ }^{2 *}$, Anamari Viegas de Araujo Motomiya ${ }^{3}$, Cristielle König Marin ${ }^{2}$, Zanandra Boff de Oliveira $^{2}$,

${ }^{1}$ CPA Consultoria e Planejamento em Agronegócio, 79824-130, Dourados/MS, Brasil.

${ }^{2}$ Campus de Cachoeira do Sul, Universidade Federal de Santa Maria, 96503-205, Cachoeira do Sul/RS, Brasil.

3 Faculdade de Ciências Agrárias, Universidade Federal da Grande Dourados, 79825-070, Dourados/MS, Brasil.

*E-mail: eduardo.bottega@ufsm.br

Recebido em: 04/08/2020

Aceito em: 29/12/2020

DOI: 10.17058/tecnolog.v25i1.15548

\section{RESUMO}

A agricultura nacional tem passado por intensas mudanças, tanto no modo de gestão das propriedades quanto no nível tecnológico empregado nas mesmas. Dentre as tecnologias adotadas, dá-se destaque à agricultura de precisão, principalmente utilizada para a aplicação de fertilizantes em taxas variadas, utilizando as informações das análises de amostras de solo, coletadas em grades contínuas georreferenciadas. O presente estudo foi conduzido em um talhão da Fazenda Planalto, localizada no município de Maracaju - MS, com o objetivo de compreender os diferentes arranjos amostrais adotados para coleta de solo e sua influência na análise geoestatística do fósforo, do potássio, da saturação por bases, do pH, da matéria orgânica e do teor de argila, bem como a confiabilidade dos mapas de predição elaborados a partir da análise geoestatística. Foram utilizados diferentes números de amostras simples para formar a amostra composta $(1,5,10,17$ e 25) combinados com um desbaste dos pontos amostrais para formar diferentes resoluções de grades (187, 95, 47 e 23 pontos). Concluiu-se que são necessárias oito amostras simples para um menor erro de predição de valores em locais não amostrados. Para estimar a dependência espacial necessita-se de grades amostrais com: 187 pontos para o fósforo potássio e saturação por bases, 95 pontos para o pH e a matéria orgânica e 23 pontos para o teor de argila. As melhores estimativas em locais não amostrados são obtidas para a argila.

Palavras-chave: Agricultura de precisão. Amostragem. Grade amostral. Variabilidade espacial.

TECNO-LÓGICA, Santa Cruz do Sul, v. 25, n. 1, p. 145-172, jul./dez. $2021 \quad \begin{aligned} & \text { A matéria publicada nesse periódico é licenciada sob forma de uma } \\ & \text { Licença Creative Commons - Atribuição } 4.0 \text { Internacional }\end{aligned}$ http://creativecommons.org/licenses/by/4.0/ 


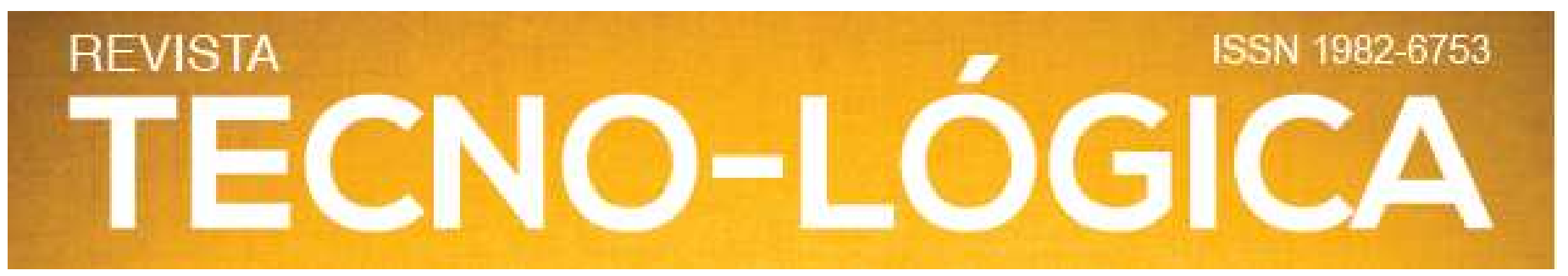

\section{Introdução}

No Brasil, a agricultura de precisão surgiu no final do século XX e desde então vem ganhando espaço como prática agronômica, adotada por um número cada vez maior de extensionistas e produtores rurais. Sua principal forma de utilização consiste no manejo da fertilidade do solo por meio de sua amostragem georreferenciada e da aplicação de corretivos e fertilizantes à taxa variável, visando a uniformização de seus atributos químicos.

A variabilidade espacial dos atributos do solo pode ser influenciada por fatores intrínsecos (material de origem, relevo, clima, organismos e tempo) e extrínsecos, normalmente relacionados com as práticas de manejo, como adubação e calagem [1], sendo verificada a heterogeneidade vertical e/ou horizontal no solo [2].

Para a amostragem do solo, normalmente são utilizadas malhas ou grades amostrais regulares [3]. A grade amostral é caracterizada por um conjunto de pontos de amostragem, separados equidistantes, sendo as coletas realizadas no entorno deste. Maior será a densidade da malha quanto menor for a distância entre os pontos.

A distância entre pontos que compõem a grade de amostragem deve ser tanto menor quanto maior for a variabilidade do solo. Nanni et al [4], estudando resoluções de grades amostrais (1,00 a 7,30 ha), concluíram que a malha amostral composta por um ponto por hectare não foi suficiente para capturar as variações do fósforo, do potássio e da saturação por bases em talhão cultivado com cana-de-açúcar. Para a determinação da matéria orgânica e do teor de argila do solo, estes autores sugerem que podem ser adotadas malhas amostrais de 2,07 e 2,88 ha, respectivamente.

Caon e Genú [5], estudando diferentes malhas amostrais $(0,25$ a 4,00 ha) através do índice Kappa, concluíram que a grade de um ponto por hectare é a mais recomendada para a elaboração de mapas dos atributos químicos de um Latossolo Bruno sob plantio direto.

Siqueira et al. [6], comparando malhas amostrais variando de 2,50 a 9,00 ha, através da raiz quadrada do erro do quadrado médio, consideraram uma malha amostral de 7,00 hectares suficiente para diagnosticar a variabilidade horizontal da saturação por bases e do teor de argila em área de cana-deaçúcar cultivada em solos classificados como Latossolo Vermelho Eutroférrico, Latossolo Vermelho Distroférrico, Latossolo Vermelho Distrófico e Latossolo Vermelho-Amarelo Distrófico.

Além da intensidade amostral, outro fator que deve ser considerado é a sua representatividade. Guarçoni et al. [7], estudando o número de amostras simples que devem ser

TECNO-LÓGICA, Santa Cruz do Sul, v. 25, n. 1, p. 145-172, jul./dez. 2021 A matéria publicada nesse periódico é licenciada sob forma de uma 


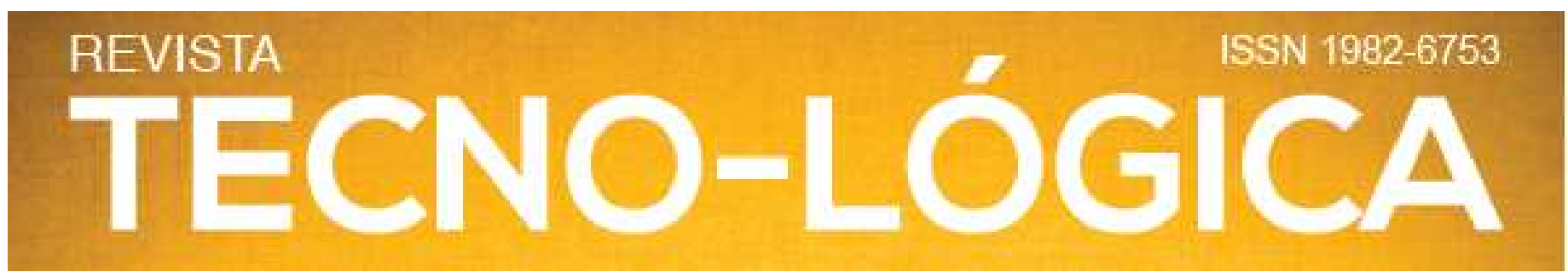

coletados para a formação de uma amostra composta 2 Parte Experimental ou Metodologia

representativa, concluíram que devem ser coletadas 25 amostras

\section{1 Área de estudo}

para se caracterizar o solo, num hexágono de $68,75 \mathrm{~cm}$ de lado e área de 1,228 $\mathrm{m}^{2}$. Schlindwein e Anguinoni [8], estudando a variabilidade horizontal do solo no sistema de plantio direto manejado pela agricultura tradicional, comentaram que o número de 20 amostras simples para a formação da amostra composta pode ser adotado, desde que seja admitido um erro de $20 \%$ em relação à média.

Desta forma, o conhecimento da influência do número de amostras simples para formar a amostra composta na qualidade dos mapas de predição é de imprescindível importância, visto que na literatura são encontrados diversos trabalhos abordando a intensidade amostral, mas são poucos os que tratam do número de amostras simples na qualidade da predição dos mapas de atributos químicos do solo.

Diante do exposto, o presente estudo objetivou avaliar o efeito de diferentes arranjos amostrais, obtidos pela variação da densidade da amostra (número de pontos) e da quantidade de amostras simples de solo, na caracterização da variabilidade espacial de fósforo $(\mathrm{P})$, de potássio $(\mathrm{K})$, da acidez do solo $(\mathrm{pH})$, da matéria orgânica (MO) e do teor de argila, em sistema de plantio direto.

A coleta de dados foi realizada na Fazenda Planalto, localizada no município de Maracaju - MS, com coordenadas geográficas de $21^{\circ} 38^{\prime} 20^{\prime}$ ' $\mathrm{S}$ e $55^{\circ} 33^{\prime} 30^{\prime}$ ' $\mathrm{W}$, entre os dias 22 e 29 de agosto de 2012. O clima da região é classificado com Am, segundo a classificação de Köppen [9], apresentando estação chuvosa no verão e estação seca no inverno, com temperatura média do mês mais frio superior a $18^{\circ} \mathrm{C}$, com precipitação média anual de 1500 a $1700 \mathrm{~mm}$ e temperatura média anual de $22,3^{\circ} \mathrm{C}$.

O estudo foi realizado em um talhão de 101 ha. O talhão é cultivado há 35 anos sob condições de sequeiro, sendo 20 anos sob semeadura direta e os últimos cinco anos sob sucessão de soja / milho safrinha + braquiária (Urochloa ruziziensis). As adubações com fósforo foram realizadas no sulco de semeadura da soja e as de potássio em cobertura, em área total na cultura do milho safrinha. O solo da área é classificado como Latossolo Vermelho Distroférrico, de textura argilosa a muito argilosa com os componentes granulométricos do solo variando de 225 a 400 $\mathrm{g} \mathrm{kg}^{-1}$ de areia, 125 a $225 \mathrm{~g} \mathrm{~kg}^{-1}$ de silte e 450 a $650 \mathrm{~g} \mathrm{~kg}^{-1}$ de argila. A altitude média em relação ao nível do mar é de $550 \mathrm{~m}$ e o relevo da área é classificado como plano. 


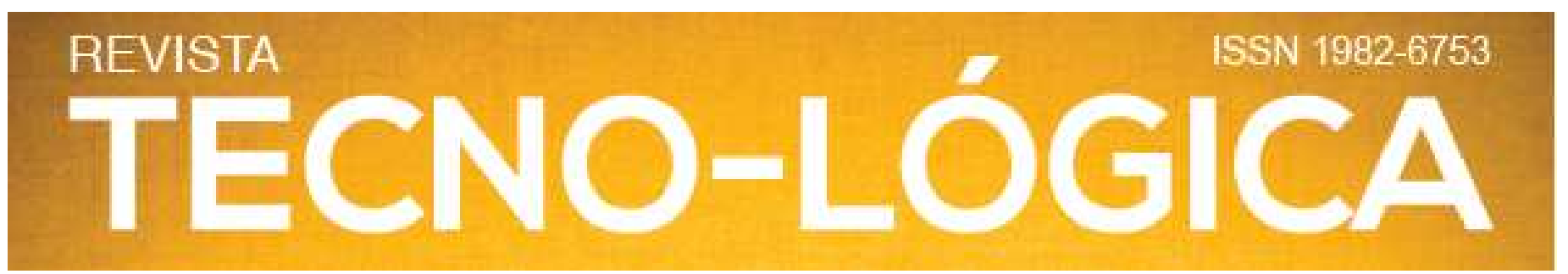

\subsection{Coleta de dados}

Para o mapeamento dos atributos químicos e físicos do solo, foi utilizada uma grade contendo 187 pontos amostrais. Os pontos foram georeferenciados utilizando-se um aparelho receptor GPS Garmin, Modelo HCx com precisão de 3,00 m.

De cada ponto amostral foram retiradas 25 amostras simples na camada de $0,0-0,20 \mathrm{~m}$ na entrelinha da cultura antecessora. Tais amostras foram coletadas aleatoriamente em um raio de $10 \mathrm{~m}$ do ponto georreferenciado e acondicionadas individualmente em embalagem plástica identificada. Para tanto, utilizou-se uma broca helicoidal de uma polegada de diâmetro acoplada a uma furadeira.

\subsection{Análises laboratoriais}

Os atributos químicos do solo avaliados foram: $\mathrm{pH}$ em $\mathrm{CaCl}_{2}(0,01 \mathrm{~mol} \mathrm{~L}-1)$, matéria orgânica $(\mathrm{MO})$, potássio $\left(\mathrm{K}^{+}\right)$, cálcio $\left(\mathrm{Ca}^{+2}\right)$, magnésio $\left(\mathrm{Mg}^{+2}\right)$, fósforo $(\mathrm{P})$ e hidrogênio mais alumínio $\left(\mathrm{H}^{+}+\mathrm{Al}^{+3}\right)$, determinados de acordo com Claessen [10]; os valores de soma de bases (SB), dada pelo somatório das bases trocáveis no solo; a capacidade de troca de cátions do solo (T), calculada pela expressão: $\mathrm{T}=\mathrm{SB}+\left(\mathrm{H}^{+}+\mathrm{Al}^{+3}\right)$; a saturação por bases (V), calculada pela expressão: $\mathrm{V}=100 \mathrm{x}$ SB/T. A composição granulométrica foi determinada por meio da dispersão com $\mathrm{NaOH}\left(0,1 \mathrm{~mol} \mathrm{~L}^{-1}\right)$ e agitação lenta durante 16 horas, sendo o teor de argila obtido pelo método da pipeta.

\subsection{Análise dos dados}

Os dados foram submetidos a uma análise exploratória para verificar a presença de valores discrepantes, sendo utilizada a metodologia proposta por Libardi et al. [11], segundo a qual o limite crítico para estes valores é definido a partir da dispersão interquartil (DQ), sendo o limite superior definido por (Q3 + 1,5 x DQ) e o inferior por (Q1 - 1,5 x DQ), em que Q1 e Q3 são o primeiro e o terceiro quartil, respectivamente. Os valores discrepantes foram excluídos do conjunto de dados.

Para a avaliação da variabilidade dos atributos químicos e físicos em função da resolução da grade amostral, a partir da grade com 187 pontos ( $0,54 \mathrm{ha})$, foram retirados pontos amostrais do banco de dados e então criadas novas grades amostrais com 95, 47 e 23 pontos (1,06; 2,14 e 4,39 ha).

Para a avaliação da variabilidade dos atributos químicos em função do número de amostras simples utilizadas para representar o ponto amostral, foram obtidas amostras compostas contendo $25,17,10,5$ e 1 amostra simples da seguinte forma: a amostra composta com 25 amostras simples foi formada por porções iguais das 25 amostras simples coletadas; a amostra composta contendo 17 amostras simples foi

TECNO-LÓGICA, Santa Cruz do Sul, v. 25, n. 1, p. 145-172, jul./dez. 2021 A matéria publicada nesse periódico é licenciada sob forma de uma 


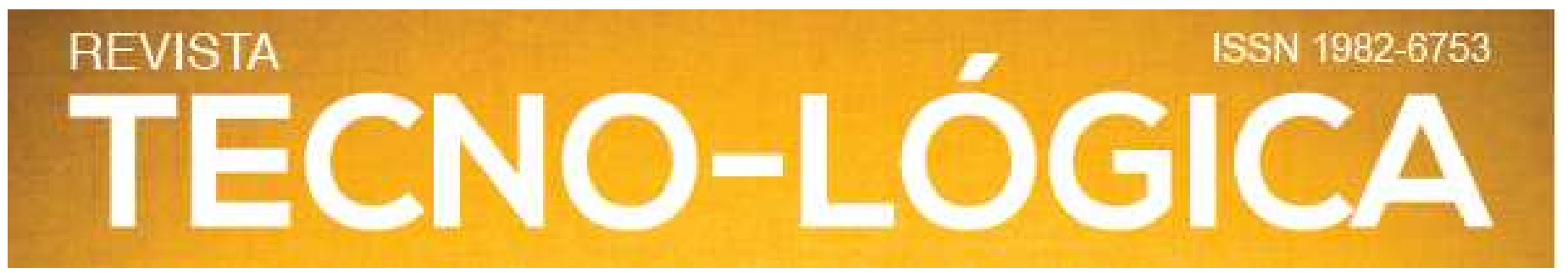

formada com porções iguais de 17 amostras simples $\left.\left(\mathrm{C}_{0}+\mathrm{C}\right)\right) \times 100$, sendo $\mathrm{C}$ o valor da variância estrutural e $\mathrm{C}_{0} \mathrm{o}$ remanescentes, após a exclusão de 8 amostras simples do ponto amostral, e assim procedeu-se até restar uma amostra simples por ponto. Para a argila só foi analisado a amostra composta contendo 25 amostras simples em função da pequena disponibilidade de solo.

A dependência espacial foi avaliada pelo método dos momentos, de Matheron. Os dados experimentais foram submetidos ao ajuste de modelos teóricos de semivariogramas, utilizando o programa GS+ [12]. Os modelos testados foram o esférico, o exponencial e o gaussiano. A escolha do modelo teve por base o maior coeficiente de determinação $\left(\mathrm{R}^{2}\right)$, na menor soma de quadrados do resíduo (SQR).

O ajuste dos modelos e a precisão da interpolação foram analisados pelos critérios da retirada de um valor amostrado e da obtenção do valor estimado do mesmo pela krigagem ordinária, utilizando-se os valores dos pontos vizinhos, sendo realizado esse processo para todos os pontos amostrados. Ao final, para cada valor verdadeiro existirá um estimado e, portanto, o erro de estimação. Os parâmetros desta validação cruzada são o coeficiente de regressão, intercepto, erro quadrático e coeficiente de determinação.

O índice de dependência espacial dos atributos (IDE) foi determinado e classificado utilizando a equação IDE $=(\mathrm{C} /$

valor do efeito pepita, assumindo os seguintes intervalos: dependência espacial baixa para IDE $<25 \%$, moderada para $25 \% \leq \mathrm{IDE} \leq 75 \%$ e forte para IDE $>75 \%$ [13].

A precisão da interpolação também foi avaliada pelo cálculo da raiz quadrada do erro médio e quadrado médio do erro [14], [15-16]. Foram usados 16 vizinhos no processo de interpolação, valor intermediário entre 4 e 20 [14]. Foi considerado também como raio de busca, valor equivalente a $80 \%$ do alcance obtido no ajuste do semivariograma teórico à semivariância empírica dos dados.

A similaridade entre os mapas foi avaliada por meio da correlação entre os valores dos 40.447 pixels dos mapas interpolados pela krigagem, comparando o mapa gerado a partir do arranjo amostral com melhor ajuste do variograma e validação cruzada com o restante dos arranjos para cada variável testada, considerando-se a classificação de Mukaka [17]. A acurácia dos mapas foi avaliada pelo RRMSE (relative root mean square error) calculado a partir dos valores observados e estimados para cada ponto amostral [18].

Foi realizada a análise de variância para avaliar o efeito do número de amostras simples na precisão da interpolação dos dados, verificando-se a significância pelo teste $\mathrm{F}$ a $5 \%$ de probabilidade e, quando significativo, foram ajustadas equações

TECNO-LÓGICA, Santa Cruz do Sul, v. 25, n. 1, p. 145-172, jul./dez. 2021 A matéria publicada nesse periódico é licenciada sob forma de uma 


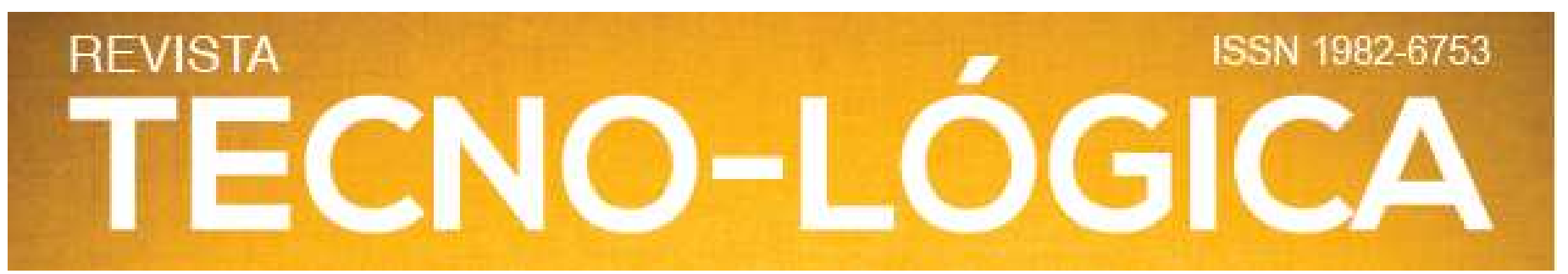

de regressão, para estudar o efeito do número de amostras no coeficiente de determinação $\left(\mathrm{R}^{2}\right)$ da validação cruzada, na raiz quadrada do erro médio (RQEM) e no erro médio percentual (EMP). Para estes procedimentos estatísticos, utilizou-se o aplicativo computacional SISVAR 5.4 [19].

\section{Resultados e discussões}

Na Tabela 1 são apresentados os parâmetros da estatística descritiva dos atributos de solo, considerando amostragem nos 187 pontos. Os valores da média e mediana foram próximos em todos os atributos, indicando que os dados tendem a uma distribuição normal. Os coeficientes de variação (CV) dos atributos estudados variaram de 4,27 para o $\mathrm{pH}$ do solo, utilizando 17 amostras simples, a 50,4\% para os teores de $\mathrm{P}$ do solo utilizando uma amostra simples por ponto. Segundo a classificação proposta por Wilding e Drees [20], os teores de P em todos os números de amostras testadas e o de $\mathrm{K}$ com uma amostra simples foram classificados como alto (CV>35\%).

Para o K, utilizando-se 5 ou mais amostras simples e para a Matéria Orgânica do solo (MO) utilizando-se 1, 5 e 25 amostras simples, o $\mathrm{CV}$ foi classificado como moderado $(15 \%<\mathrm{CV}<35 \%)$, e, para o restante do número de amostras testados para a M.O. e todos os números de amostras testados para o $\mathrm{V} \%$ e o $\mathrm{pH}$, o $\mathrm{CV}$ foi classificado como baixo $(\mathrm{CV}<15 \%)$.
Destaca-se que, para a M.O. do solo, os coeficientes de variação foram muito próximos entre si e sem influência evidente do número de amostras utilizadas, oscilando pouco acima ou pouco abaixo de $15 \%$, o que resultou na classificação entre baixo e moderado.

Para os demais atributos, observou-se uma tendência de aumento do coeficiente de variação conforme se diminui o número de amostras. Provavelmente, o aumento da dispersão observado para o fósforo e o potássio, quando se utiliza menor número de amostras simples por ponto, deve-se a aplicações sucessivas de fertilizantes no sulco de semeadura, fazendo com que ocorram grandes variações nos teores dos nutrientes a curtas distâncias, principalmente no caso do fósforo, pouco móvel no solo. Os valores médios dos atributos do solo não sofreram grandes variações em função do número de amostras utilizadas e foram classificados como altos, segundo a Embrapa [21].

Na Tabela 2 são apresentados os parâmetros ajustados à variância empírica dos valores de fósforo, considerando os diferentes arranjos amostrais estudados. Para os teores de P no solo, foram ajustados semivariogramas em todos os arranjos utilizados, exceto, para o com 17 amostras simples e 23 pontos amostrais, no qual observou-se efeito pepita puro. Dependendo do arranjo amostral, ocorreu mudança dos modelos teóricos ajustados, demonstrando que houve grande influência do arranjo amostral na análise geoestatística dos dados. 


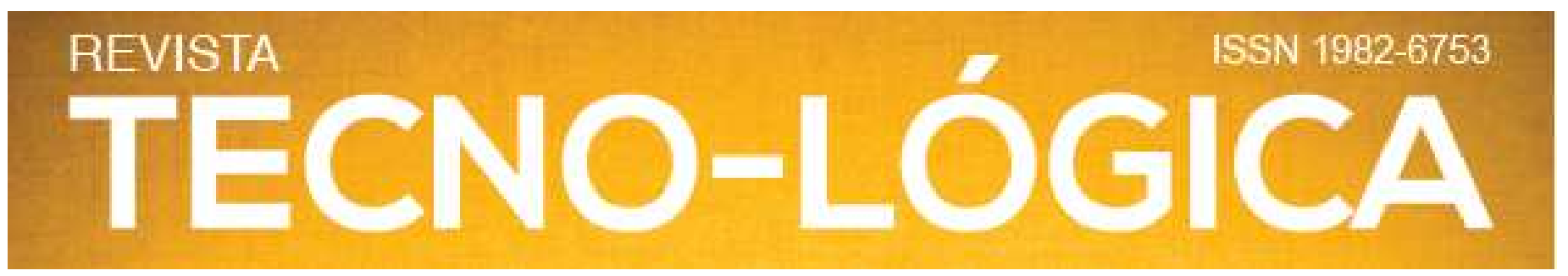

Tabela 1. Estatística descritiva dos atributos do solo na malha amostral de 187 pontos

\begin{tabular}{|c|c|c|c|c|c|c|c|c|c|}
\hline Amostras & Atributo & Média & Mediana & Min. & Máx. & $\mathbf{S}^{(7)}$ & $\mathrm{CV}^{(8)}(\%)$ & $\overline{Q I^{(9)}}$ & $\overline{\mathbf{Q S}^{(10)}}$ \\
\hline $25 \mathrm{~A}^{(1)}$ & $\mathrm{P}^{(2)}$ & 12,69 & 12,08 & 3,97 & 28,32 & 4,85 & 38,23 & 8,91 & 15,54 \\
\hline $17 \mathrm{~A}$ & $\mathrm{P}$ & 11,74 & 11,08 & 3,59 & 27,67 & 4,35 & 37,03 & 8,35 & 14,51 \\
\hline $10 \mathrm{~A}$ & $\mathrm{P}$ & 11,16 & 10,03 & 2,85 & 30,93 & 4,49 & 40,25 & 7,79 & 13,58 \\
\hline $5 \mathrm{~A}$ & $\mathrm{P}$ & 10,23 & 9,10 & 2,47 & 26,45 & 4,52 & 44,18 & 6,81 & 12,18 \\
\hline $1 \mathrm{~A}$ & $\mathrm{P}$ & 11,28 & 10,08 & 2,85 & 30,18 & 5,69 & 50,40 & 7,07 & 14,84 \\
\hline $25 \mathrm{~A}$ & $\mathrm{~K}^{(3)}$ & 0,48 & 0,47 & 0,23 & 0,89 & 0,13 & 26,19 & 0,39 & 0,56 \\
\hline $17 \mathrm{~A}$ & $\mathrm{~K}$ & 0,47 & 0,45 & 0,18 & 0,82 & 0,13 & 27,33 & 0,37 & 0,55 \\
\hline $10 \mathrm{~A}$ & $\mathrm{~K}$ & 0,45 & 0,43 & 0,17 & 0,90 & 0,13 & 28,25 & 0,36 & 0,52 \\
\hline $5 \mathrm{~A}$ & $\mathrm{~K}$ & 0,44 & 0,42 & 0,18 & 0,93 & 0,13 & 30,96 & 0,34 & 0,51 \\
\hline $1 \mathrm{~A}$ & K & 0,44 & 0,41 & 0,14 & 1,01 & 0,16 & 36,40 & 0,33 & 0,53 \\
\hline $25 \mathrm{~A}$ & $\mathrm{~V}^{(4)}$ & 68,46 & 68,89 & 53,56 & 83,35 & 5,67 & 8,28 & 64,97 & 72,43 \\
\hline $17 \mathrm{~A}$ & $\mathrm{~V}$ & 66,41 & 66,17 & 51,38 & 80,45 & 5,46 & 8,21 & 62,90 & 70,36 \\
\hline $10 \mathrm{~A}$ & $\mathrm{~V}$ & 66,17 & 66,00 & 50,07 & 81,59 & 6,10 & 9,22 & 62,86 & 70,54 \\
\hline $5 \mathrm{~A}$ & $\mathrm{~V}$ & 66,94 & 67,67 & 50,45 & 82,50 & 6,38 & 9,54 & 62,42 & 71,03 \\
\hline $1 \mathrm{~A}$ & $\mathrm{~V}$ & 67,08 & 66,82 & 50,72 & 82,85 & 6,63 & 9,88 & 63,21 & 72,12 \\
\hline $25 \mathrm{~A}$ & $\mathrm{pH}^{(5)}$ & 5,19 & 5,20 & 4,60 & 5,78 & 0,22 & 4,29 & 5,04 & 5,34 \\
\hline $17 \mathrm{~A}$ & $\mathrm{pH}$ & 5,21 & 5,21 & 4,60 & 5,76 & 0,22 & 4,27 & 5,06 & 5,36 \\
\hline $10 \mathrm{~A}$ & $\mathrm{pH}$ & 5,19 & 5,16 & 4,56 & 5,75 & 0,23 & 4,36 & 5,03 & 5,32 \\
\hline $5 \mathrm{~A}$ & $\mathrm{pH}$ & 5,18 & 5,17 & 4,60 & 5,76 & 0,24 & 4,57 & 5,04 & 5,34 \\
\hline $1 \mathrm{~A}$ & $\mathrm{pH}$ & 5,21 & 5,20 & 4,40 & 6,05 & 0,31 & 6,03 & 5,00 & 5,43 \\
\hline $25 \mathrm{~A}$ & $\mathrm{MO}^{(6)}$ & 29,44 & 29,10 & 18,35 & 39,81 & 4,57 & 15,53 & 26,45 & 32,55 \\
\hline $17 \mathrm{~A}$ & MO & 29,45 & 29,28 & 18,66 & 40,01 & 4,36 & 14,81 & 26,57 & 32,20 \\
\hline $10 \mathrm{~A}$ & MO & 29,05 & 28,40 & 20,21 & 41,03 & 4,02 & 13,83 & 26,05 & 31,71 \\
\hline $5 \mathrm{~A}$ & MO & 28,60 & 28,01 & 18,47 & 39,94 & 4,40 & 15,38 & 25,82 & 31,12 \\
\hline $1 \mathrm{~A}$ & MO & 28,65 & 27,98 & 17,90 & 42,04 & 4,77 & 16,66 & 25,07 & 31,86 \\
\hline $25 \mathrm{~A}$ & Argila & 540,11 & 550,00 & 450,00 & 650,00 & 62,88 & 11,64 & 500,00 & 600,00 \\
\hline
\end{tabular}

${ }^{(1)}$ Número de amostras simples; ${ }^{(2)}$ Teor de fósforo $\left(\mathrm{mg} \mathrm{dm}^{-3}\right) ;{ }^{(3)}$ Teor de potássio $\left(\mathrm{cmol}_{\mathrm{c}} \mathrm{dm}^{-3}\right) ;{ }^{(4)}$ Saturação por bases $(\%)$; ${ }^{(5)} \mathrm{pH}$ do solo em CaCl ${ }_{2}{ }^{(6)} \mathrm{Teor}$ de matéria orgânica $\left(\mathrm{g} \mathrm{kg}^{-1}\right)$.

Os valores do alcance do $\mathrm{P}$ variaram de $154 \mathrm{~m}(25$ amostras simples e 187 pontos) a $2263 \mathrm{~m}$ (5 amostras simples e 23 pontos). Em todos os arranjos amostrais mais densos (187 pontos), foram observados os melhores ajustes do variograma, exceto no arranjo formado por apenas uma amostra simples por ponto. Nesse caso, verificou-se a redução do coeficiente de determinação e o aumento pronunciado da SQR. A perda de qualidade dos ajustes dos semivariogramas nos arranjos com menor número de pontos amostrais é explicada pelo aumento da distância entre as amostras e também pela diminuição do número de amostras utilizadas na construção de semivariogramas pelo método dos momentos de Matheron [16, 22].

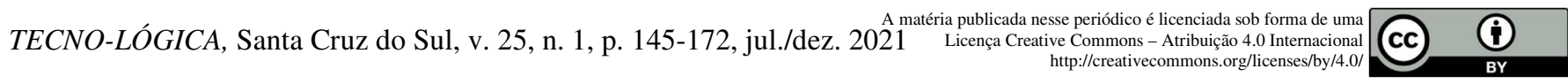
151 


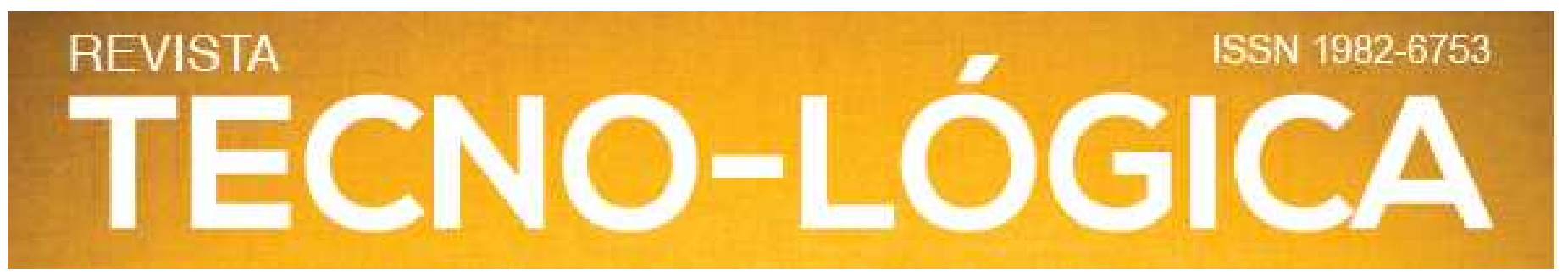

A dependência espacial foi classificada como moderada

a forte para todos os arranjos amostrais que apresentaram

dependência espacial. Diferente do observado por Souza et al.

[23], a diminuição do número de pontos amostrais não ocasionou aumento evidente do efeito pepita, entretanto, a redução do número de amostras simples demonstrou aumentar tal efeito nos modelos, indicando a existência de grandes variações do teor do nutriente do solo a curtas distâncias [7].

Tabela 2. Parâmetros dos modelos teóricos dos semivariogramas ajustados para os teores de $\mathrm{P}\left(\mathrm{mg} \mathrm{dm}^{-3}\right)$ do solo em função dos diferentes arranjos amostrais utilizados

\begin{tabular}{|c|c|c|c|c|c|c|c|c|}
\hline Arranjo & Pontos excluídos & Modelo & Alcance (m) & $\mathrm{C}_{0}+\mathrm{C}^{(3)}$ & $\mathbf{C}_{0}^{(4)}$ & IDE $^{(5)}$ & $\mathbf{S Q R}^{(6)}$ & $\mathbf{R}^{2(7)}$ \\
\hline $25 \mathrm{~A}^{(1)} 187 \mathrm{Pt}^{(2)}$ & 2 & $\operatorname{EXP}^{(8)}$ & 154 & 23,22 & 7,90 & 66 & 2,1 & 0,98 \\
\hline 17 A $187 \mathrm{Pt}$ & 3 & EXP & 309 & 20,93 & 8,22 & 60 & 0,8 & 0,99 \\
\hline $10 \mathrm{~A} 187 \mathrm{Pt}$ & 4 & EXP & 500 & 26,02 & 10,72 & 58 & 1,6 & 0,98 \\
\hline $5 \mathrm{~A} 187 \mathrm{Pt}$ & 2 & ESF & 406 & 19,22 & 8,21 & 57 & 1,4 & 0,98 \\
\hline $1 \mathrm{~A} 187 \mathrm{Pt}$ & 3 & EXP & 192 & 34.53 & 17,26 & 50 & 23,2 & 0,83 \\
\hline $25 \mathrm{~A} 95 \mathrm{Pt}$ & 0 & EXP & 187 & 25,03 & 12,51 & 50 & 40,0 & 0,52 \\
\hline 17 A $95 \mathrm{Pt}$ & 1 & $\mathrm{GAU}^{(9)}$ & 1251 & 41,99 & 11,00 & 73 & 20,8 & 0,88 \\
\hline 10 A $95 \mathrm{Pt}$ & 2 & EXP & 246 & 18,11 & 6,85 & 62 & 9,6 & 0,85 \\
\hline $5 \mathrm{~A} 95 \mathrm{Pt}$ & 0 & GAU & 1148 & 47,40 & 13,70 & 71 & 10,7 & 0,93 \\
\hline $1 \mathrm{~A} 95 \mathrm{Pt}$ & 1 & EXP & 721 & 40,95 & 20,47 & 50 & 15,4 & 0,86 \\
\hline $25 \mathrm{~A} 47 \mathrm{Pt}$ & 0 & GAU & 1378 & 68,20 & 13,60 & 80 & 80,7 & 0,91 \\
\hline $17 \mathrm{~A} 47 \mathrm{Pt}$ & 1 & GAU & 1205 & 36,01 & 7,51 & 79 & 5,8 & 0,96 \\
\hline $10 \mathrm{~A} 47 \mathrm{Pt}$ & 0 & GAU & 1202 & 48,00 & 8,50 & 82 & 22,0 & 0,93 \\
\hline $5 \mathrm{~A} 47 \mathrm{Pt}$ & 0 & GAU & 1131 & 51,20 & 10,60 & 79 & 4,6 & 0,98 \\
\hline $1 \mathrm{~A} 47 \mathrm{Pt}$ & 0 & $\operatorname{ESF}^{(10)}$ & 726 & 34,14 & 14,95 & 56 & 34,0 & 0,84 \\
\hline 25 A $23 \mathrm{Pt}$ & 0 & GAU & 1357 & 40,93 & 9,97 & 75 & 44,3 & 0,73 \\
\hline 17 A $23 \mathrm{Pt}$ & 0 & $\mathrm{EPP}^{(11)}$ & - & 17,98 & 17,98 & - & - & - \\
\hline 10 A $23 \mathrm{Pt}$ & 0 & GAU & 1125 & 33,90 & 6,74 & 80 & 11,2 & 0,92 \\
\hline $5 \mathrm{~A} 23 \mathrm{Pt}$ & 0 & EXP & 2263 & 41,92 & 11,23 & 73 & 10,9 & 0,79 \\
\hline $1 \mathrm{~A} 23 \mathrm{Pt}$ & 0 & ESF & 321 & 30,22 & 0,01 & 100 & 30,4 & 0,12 \\
\hline
\end{tabular}

Pela validação cruzada dos modelos (Tabela 3), confirma-se que as melhores estimativas foram obtidas pela malha mais densa, combinada com pelo menos 5 ou mais amostras simples para formar a amostra composta. Observou-se, ainda, que não houve melhoria da qualidade dos modelos ajustados quando se utilizou amostras compostas com mais de 17 amostras simples. Os coeficientes de determinação da validação cruzada foram baixos $\left(\mathrm{R}^{2}<0,5\right)$ para todos os arranjos amostrais, segundo a classificação adotada por Nanni et al. [4].

TECNO-LÓGICA, Santa Cruz do Sul, v 25, n. 1, p. 145-172,jul./dez. 2021 A matéria publicada nesse periódico é licenciada sob forma de uma 


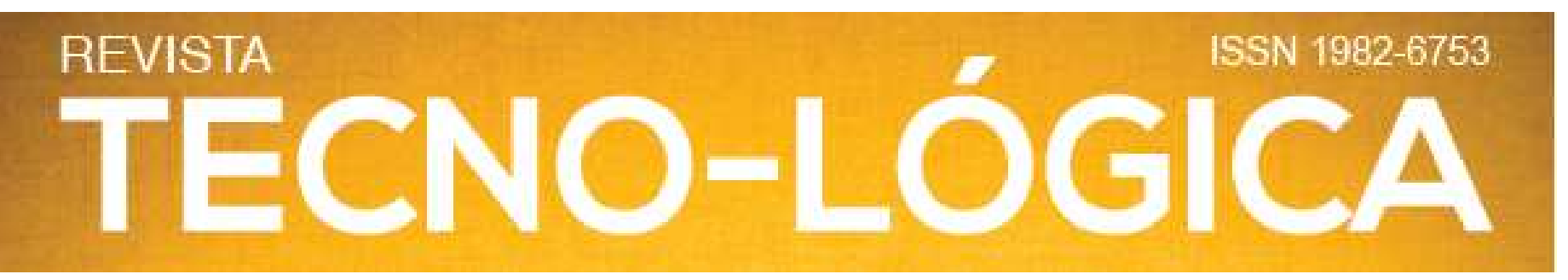

Tabela 3. Validação cruzada dos modelos teóricos dos semivariogramas ajustados para os teores de $\mathrm{P}\left(\mathrm{mg} \mathrm{dm}^{-3}\right)$ do solo nos diferentes arranjos amostrais utilizados

\begin{tabular}{|c|c|c|c|c|c|c|}
\hline Arranjo & Coeficiente de regressão & Intercepto $(Y)$ & Erro padrão (SE) & $\mathbf{R}^{2(3)}$ & RQEM $^{(4)}$ & $\mathbf{E M P}^{(5)}$ \\
\hline $25 \mathrm{~A}^{(1)} 187 \mathrm{Pt}^{(2}$ & 0,949 & 0,64 & 0,097 & 0,34 & 3,923 & 27,46 \\
\hline 17 A $187 \mathrm{Pt}$ & 0,958 & 0,47 & 0,095 & 0,36 & 3,469 & 26,48 \\
\hline 10 A $187 \mathrm{Pt}$ & 0,954 & 0,48 & 0,105 & 0,31 & 3,718 & 29,47 \\
\hline $5 \mathrm{~A} 187 \mathrm{Pt}$ & 0,946 & 0,54 & 0,093 & 0,36 & 3,608 & 32,10 \\
\hline $1 \mathrm{~A} 187 \mathrm{Pt}$ & 0,717 & 3,19 & 0,143 & 0,12 & 5,370 & 47,40 \\
\hline 25 A $95 \mathrm{Pt}$ & 0,657 & 4,31 & 0,170 & 0,14 & 4,665 & 34,48 \\
\hline 17 A $95 \mathrm{Pt}$ & 0,977 & 0,25 & 0,149 & 0,32 & 3,400 & 28,07 \\
\hline $10 \mathrm{~A} 95 \mathrm{Pt}$ & 0,833 & 1,78 & 0,155 & 0,24 & 3,689 & 31,50 \\
\hline $5 \mathrm{~A} 95 \mathrm{Pt}$ & 0,921 & 0,80 & 0,159 & 0,26 & 3,941 & 38,69 \\
\hline $1 \mathrm{~A} 95 \mathrm{Pt}$ & 0,828 & 1,92 & 0,195 & 0,16 & 5,098 & 46,55 \\
\hline $25 \mathrm{~A} 47 \mathrm{Pt}$ & 0,676 & 4,21 & 0,285 & 0,11 & 4,478 & 36,23 \\
\hline $17 \mathrm{~A} 47 \mathrm{Pt}$ & 0,878 & 1,40 & 0,206 & 0,29 & 3,160 & 27,50 \\
\hline $10 \mathrm{~A} 47 \mathrm{Pt}$ & 0,864 & 1,48 & 0,188 & 0,32 & 3,454 & 33,06 \\
\hline $5 \mathrm{~A} 47 \mathrm{Pt}$ & 0,804 & 2,13 & 0,216 & 0,24 & 3,981 & 38,42 \\
\hline $1 \mathrm{~A} 47 \mathrm{Pt}$ & 0,780 & 2,58 & 0,295 & 0,13 & 5,152 & 49,04 \\
\hline $25 \mathrm{~A} 23 \mathrm{Pt}$ & $-0,042$ & 13,57 & 0,559 & 0,00 & 4,180 & 33,78 \\
\hline $10 \mathrm{~A} 23 \mathrm{Pt}$ & 0,749 & 2,95 & 0,332 & 0,19 & 3,377 & 31,28 \\
\hline $5 \mathrm{~A} 23 \mathrm{Pt}$ & 0,470 & 6,24 & 0,506 & 0,04 & 4,268 & 32,78 \\
\hline $1 \mathrm{~A} 23 \mathrm{Pt}$ & $-0,380$ & 17,32 & 0,464 & 0,03 & 6,353 & 56,12 \\
\hline
\end{tabular}

${ }^{(1)}$ Número de amostras simples; ${ }^{(2)}$ Número de pontos amostrais; ${ }^{(3)}$ Coeficiente de determinação; ${ }^{(4)}$ Raiz quadrada do erro médio; ${ }^{(5)}$ Erro médio percentual.

Comparando o arranjo amostral com melhor ajuste do variograma e a validação cruzada para o fósforo (17 A $187 \mathrm{Pt})$ com os demais, observa-se que, quando se utiliza arranjos amostrais com menos de 10 amostras simples por ponto amostral, ocorre aumento substancial do RRMSE dos mapas, indicando perda de acurácia dos mesmos, principalmente quando se utiliza apenas uma amostra simples por ponto. A correlação entre os mapas foi muito forte $(r>0,90)$ e forte $(0,69<r<0,90)$ nas densidades amostrais de 187 pontos e 95 pontos, respectivamente (Figura 1).
Para avaliar a variabilidade espacial dos teores de K no solo, foram ajustados os modelos exponencial e esférico, dependendo do arranjo amostral utilizado (Tabela 4). Para os modelos ajustados aos teores de $\mathrm{P}$, foram observadas menores variações do alcance espacial entre os arranjos, variando de 104 m (5 amostras simples e 187 pontos) a $1268 \mathrm{~m}$ (5 amostras simples e 95 pontos). Há uma menor variação dos alcances da dependência espacial entre os modelos teóricos ajustados, quando comparado com o $\mathrm{P}$, principalmente na malha amostral mais densa (104 a 293 m). Da mesma forma como ocorreu com 


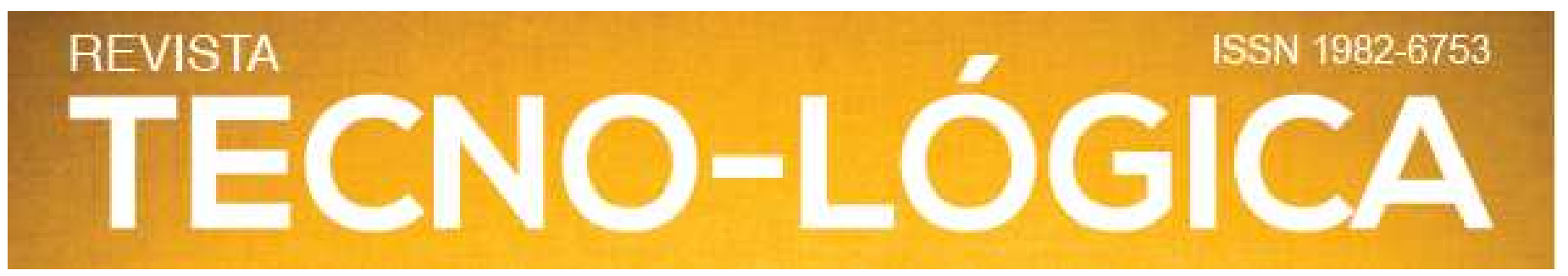

o P, quando se utilizou arranjos amostrais contendo apenas uma amostra simples por ponto, houve deterioração da qualidade dos ajustes dos modelos teóricos. O IDE para os teores de K variou de moderado a forte (50 a 100\%). Não foi observado aumento do efeito pepita conforme se distanciaram os pontos amostrais.

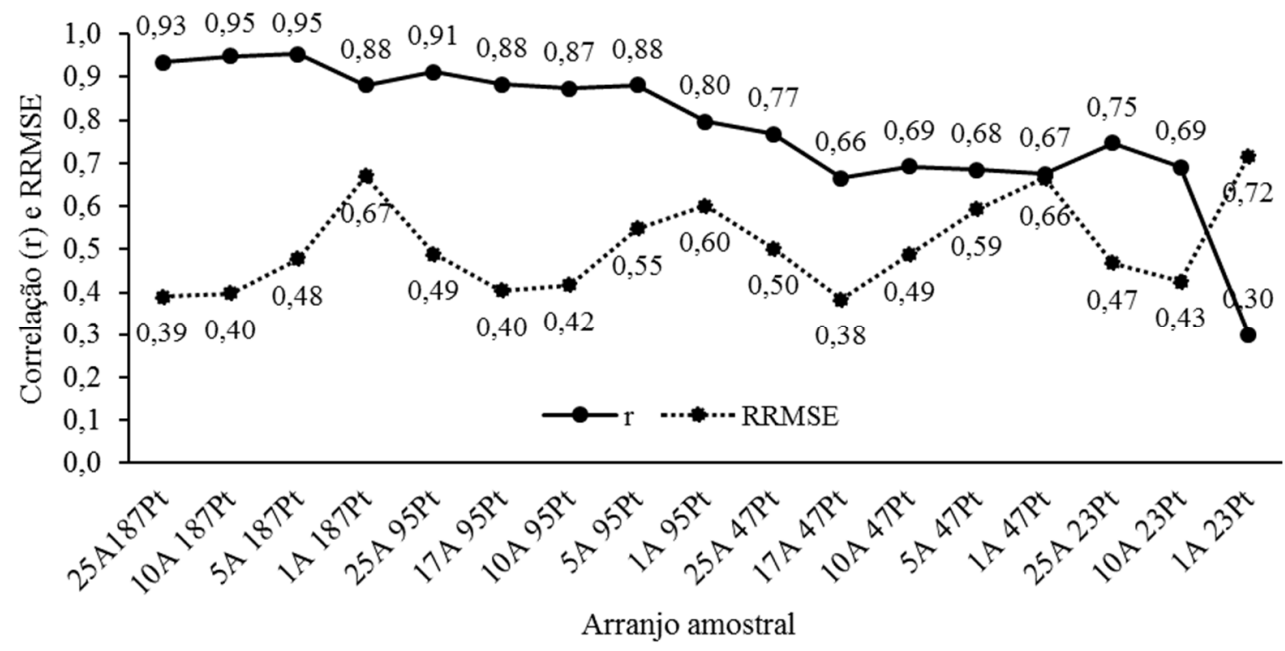

Figura 1. Correlação (r) e valores de RRMSE do mapa de variabilidade com melhor ajuste do variograma e validação cruzada para o fósforo (17A 187Pt) comparado com os mapas dos restantes arranjos amostrais.

Os parâmetros da validação cruzada também foram melhores quando se utilizou a malha amostral mais densa combinada com pelo menos 5 amostras simples por ponto, não se observando melhorias expressivas na precisão quando foram utilizadas mais de 10 amostras simples por ponto (Tabela 5). Provavelmente, a menor acurácia dos mapas com menores números de amostras simples ocorreu devido à existência de grandes variações nos teores de nutrientes a curtas distâncias, tanto para o P, distribuído no sulco de semeadura, quanto para o $\mathrm{K}$, mesmo esse nutriente sendo distribuído em superfície em área total. 


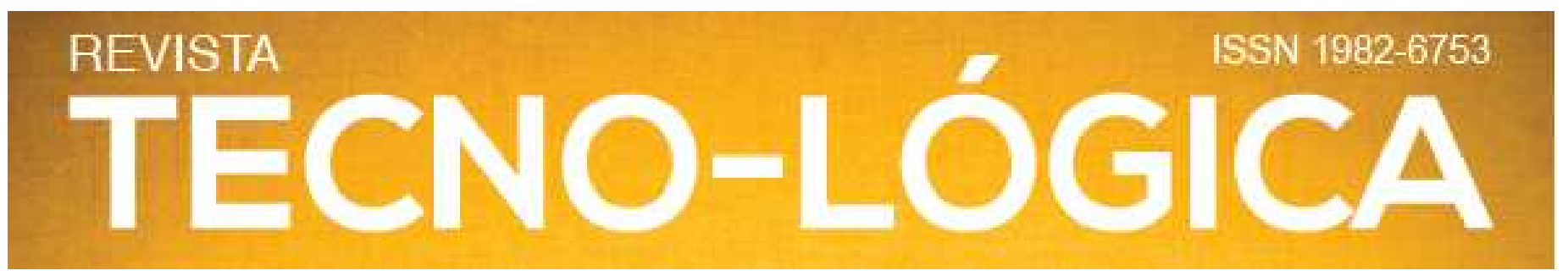

Tabela 4. Parâmetros dos modelos teóricos dos semivariogramas ajustados para os teores de $\mathrm{K}\left(\mathrm{cmol}_{\mathrm{c}} \mathrm{dm}^{-3}\right)$ no solo nos diferentes arranjos amostrais utilizados

\begin{tabular}{|c|c|c|c|c|c|c|c|c|}
\hline Arranjo & Pontos excluídos & Modelo & Alcance (m) & $\mathrm{C}_{0}+\mathrm{C}^{(3)}$ & $\mathrm{C}_{0}^{(4)}$ & IDE $^{(5)}$ & $\mathbf{S Q R}^{(\mathbf{6})}$ & $\mathbf{R}^{2(7)}$ \\
\hline $25 \mathrm{~A}^{(1)} 187 \mathrm{Pt}^{(2)}$ & 3 & $\mathrm{EXP}^{(8)}$ & 124 & 0,0211 & 0,0038 & 81 & $3,4 \times 10^{-6}$ & 0,95 \\
\hline 17 A $187 \mathrm{Pt}$ & 4 & EXP & 177 & 0,0202 & 0,0071 & 64 & $1,3 \times 10^{-6}$ & 0,97 \\
\hline 10 A $187 \mathrm{Pt}$ & 4 & EXP & 122 & 0,0201 & 0,0036 & 81 & $1,9 \times 10^{-6}$ & 0,97 \\
\hline $5 \mathrm{~A} 187 \mathrm{Pt}$ & 4 & EXP & 104 & 0,0230 & 0,0033 & 85 & $1,5 \times 10^{-6}$ & 0,98 \\
\hline $1 \mathrm{~A} 187 \mathrm{Pt}$ & 3 & ESF & 293 & 0,0290 & 0,0136 & 53 & $7,5 \times 10^{-6}$ & 0,90 \\
\hline 25 A $95 \mathrm{Pt}$ & 3 & EXP & 334 & 0,0137 & 0,0063 & 54 & $7,8 \times 10^{-6}$ & 0,79 \\
\hline 17 A $95 \mathrm{Pt}$ & 3 & EXP & 363 & 0,0127 & 0,0059 & 53 & $3,8 \times 10^{-6}$ & 0,87 \\
\hline 10 A $95 \mathrm{Pt}$ & 3 & EXP & 541 & 0,0141 & 0,0062 & 55 & $2,7 \times 10^{-6}$ & 0,91 \\
\hline 5 A $95 \mathrm{Pt}$ & 2 & EXP & 958 & 0,0175 & 0,0087 & 50 & $3,2 \times 10^{-6}$ & 0,85 \\
\hline $1 \mathrm{~A} 95 \mathrm{Pt}$ & 0 & EXP & 115 & 0,0216 & 0,0037 & 83 & $1,8 \times 10^{-5}$ & 0,86 \\
\hline $25 \mathrm{~A} 47 \mathrm{Pt}$ & 0 & $\mathrm{ESF}^{(9)}$ & 568 & 0,0142 & 0,0071 & 50 & $1,0 \times 10^{-6}$ & 0,93 \\
\hline $17 \mathrm{~A} 47 \mathrm{Pt}$ & 1 & EXP & 1081 & 0,0167 & 0,0070 & 57 & $2,8 \times 10^{-6}$ & 0,72 \\
\hline $10 \mathrm{~A} 47 \mathrm{Pt}$ & 1 & EXP & 495 & 0,0126 & 0,0027 & 78 & $7,0 \times 10^{-7}$ & 0,96 \\
\hline $5 \mathrm{~A} 47 \mathrm{Pt}$ & 1 & EXP & 259 & 0,0125 & 0,0031 & 75 & $4,0 \times 10^{-7}$ & 0,97 \\
\hline $1 \mathrm{~A} 47 \mathrm{Pt}$ & 0 & ESF & 204 & 0,0151 & 0,0004 & 97 & $2,0 \times 10^{-6}$ & 0,56 \\
\hline 25 A $23 \mathrm{Pt}$ & 0 & ESF & 443 & 0,0201 & 0,0000 & 100 & $9,9 \times 10^{-5}$ & 0,34 \\
\hline 17 A $23 \mathrm{Pt}$ & 0 & ESF & 369 & 0,0174 & 0,0002 & 98 & $2,2 \times 10^{-4}$ & 0,09 \\
\hline 10 A $23 \mathrm{Pt}$ & 0 & ESF & 377 & 0,0136 & 0,0002 & 98 & $1,0 \times 10^{-4}$ & 0,12 \\
\hline $5 \mathrm{~A} 23 \mathrm{Pt}$ & 0 & ESF & 304 & 0,0189 & 0,0001 & 99 & $1,9 \times 10^{-4}$ & 0,03 \\
\hline $1 \mathrm{~A} 23 \mathrm{Pt}$ & 0 & ESF & 300 & 0,0131 & 0,0001 & 99 & $6,6 \times 10^{-4}$ & 0,04 \\
\hline
\end{tabular}

(1) Número de amostras simples; ${ }^{(2)}$ Número de pontos amostrais; ${ }^{(3)}$ Patamar; ${ }^{(4)}$ Efeito pepita; ${ }^{(5)}$ Índice de dependência espacial; ${ }^{(6)}$ Soma de quadrados dos resíduos; ${ }^{(7)}$

Coeficiente de determinação; ${ }^{(8)}$ Exponencial; ${ }^{(9)}$ Esférico.

TECNO-LÓGICA, Santa Cruz do Sul, v. 25, n. 1, p. 145-172, jul./dez. 2021 $\begin{array}{r}\text { A matéria publicada nesse periódico é licenciada sob forma de uma } \\ \text { Licença Creative Commons - Atribuição 4.0 Internacional } \\ \text { http://creativecommons.org/licenses/by/4.0/ }\end{array}$ CC. 


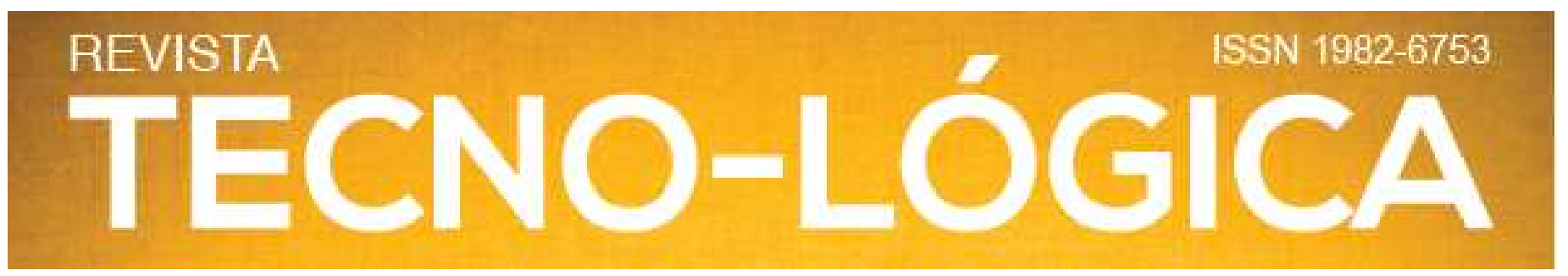

Tabela 5. Validação cruzada dos modelos teóricos dos semivariogramas ajustados para os teores de $\mathrm{K}\left(\mathrm{cmol}_{\mathrm{c}} \mathrm{dm}^{-3}\right)$ do solo nos diferentes arranjos amostrais utilizados

\begin{tabular}{|c|c|c|c|c|c|c|}
\hline Arranjo & Coeficiente de regressão & Intercepto (Y) & Erro padrão (SE) & $\mathbf{R}^{2(3)}$ & RQEM $^{(4)}$ & $\mathbf{E M P}^{(5)}$ \\
\hline $25 \mathrm{~A}^{(1)} 187 \mathrm{Pt}^{(2)}$ & 0,859 & 0,07 & 0,103 & 0,28 & 0,1150 & 19,08 \\
\hline $17 \mathrm{~A} 187 \mathrm{Pt}$ & 0,913 & 0,04 & 0,118 & 0,25 & 0,1135 & 19,78 \\
\hline $10 \mathrm{~A} 187 \mathrm{Pt}$ & 0,916 & 0,04 & 0,102 & 0,31 & 0,1099 & 19,92 \\
\hline $5 \mathrm{~A} 187 \mathrm{Pt}$ & 0,908 & 0,04 & 0,097 & 0,32 & 0,1177 & 23,57 \\
\hline $1 \mathrm{~A} 187 \mathrm{Pt}$ & 0,826 & 0,08 & 0,138 & 0,16 & 0,1521 & 30,67 \\
\hline 25 A $95 \mathrm{Pt}$ & 0,809 & 0,09 & 0,195 & 0,16 & 0,0975 & 19,01 \\
\hline 17 A $95 \mathrm{Pt}$ & 0,756 & 0,11 & 0,212 & 0,12 & 0,0972 & 19,18 \\
\hline $10 \mathrm{~A} 95 \mathrm{Pt}$ & 0,782 & 0,09 & 0,217 & 0,13 & 0,0956 & 19,93 \\
\hline $5 \mathrm{~A} 95 \mathrm{Pt}$ & 0,683 & 0,13 & 0,267 & 0,07 & 0,1060 & 23,10 \\
\hline $1 \mathrm{~A} 95 \mathrm{Pt}$ & 0,072 & 0,41 & 0,156 & 0,01 & 0,1671 & 34,69 \\
\hline $25 \mathrm{~A} 47 \mathrm{Pt}$ & 0,753 & 0,11 & 0,259 & 0,16 & 0,1022 & 19,24 \\
\hline $17 \mathrm{~A} 47 \mathrm{Pt}$ & 0,689 & 0,14 & 0,368 & 0,07 & 0,0954 & 17,92 \\
\hline $10 \mathrm{~A} 47 \mathrm{Pt}$ & 0,731 & 0,11 & 0,237 & 0,19 & 0,0862 & 16,91 \\
\hline $5 \mathrm{~A} 47 \mathrm{Pt}$ & 0,649 & 0,15 & 0,273 & 0,11 & 0,0911 & 19,55 \\
\hline $1 \mathrm{~A} 47 \mathrm{Pt}$ & 0,099 & 0,38 & 0,280 & 0,01 & 0,1333 & 28,68 \\
\hline $25 \mathrm{~A} 23 \mathrm{Pt}$ & 0,975 & 0,00 & 0,240 & 0,44 & 0,1073 & 23,32 \\
\hline $17 \mathrm{~A} 23 \mathrm{Pt}$ & 1,010 & $-0,01$ & 0,300 & 0,35 & 0,1101 & 21,86 \\
\hline 10 A $23 \mathrm{Pt}$ & 0,817 & 0,07 & 0,328 & 0,23 & 0,1058 & 22,58 \\
\hline $5 \mathrm{~A} 23 \mathrm{Pt}$ & 0,770 & 0,10 & 0,401 & 0,15 & 0,1325 & 27,58 \\
\hline $1 \mathrm{~A} 23 \mathrm{Pt}$ & 0,300 & 0,28 & 0,485 & 0,02 & 0,1227 & 28,37 \\
\hline
\end{tabular}

${ }^{(1)}$ Número de amostras simples ${ }^{(2)}$ Número de pontos amostrais; ${ }^{(3)}$ Coeficiente de determinação; ${ }^{(4)}$ Raiz quadrada do erro médio; ${ }^{(5)}$ Erro médio percentual.

Para os mapas de variabilidade do K (Figura 2), observou-se correlações muito fortes $(r>0,90)$ apenas entre os mapas que utilizaram 187 pontos amostrais, combinado com pelo menos 5 amostras simples. Os arranjos contendo 5 amostras simples, ou menos, sempre apresentaram menor acurácia dos mapas (maior RRMSE).

Para a saturação por bases do solo, observou-se menores variações nos valores do alcance espacial, sendo o modelo esférico o que mais se ajustou ao padrão de variação desse atributo químico (Tabela 6). Os alcances da dependência espacial variaram de $103 \mathrm{~m}$ (1 amostra simples e 187 pontos) a 589 m (5 amostras simples e 95 pontos).

TECNO-LÓGICA, Santa Cruz do Sul, v. 25, n. 1, p. 145-172, jul./dez. $2021 \stackrel{\text { A matéria publicada nesse periódico é licenciada sob forma de uma }}{\text { Licença Creative Commons - Atribuição } 4.0 \text { Internacional }}$ http://creativecommons.org/licenses/by/4.0/ 

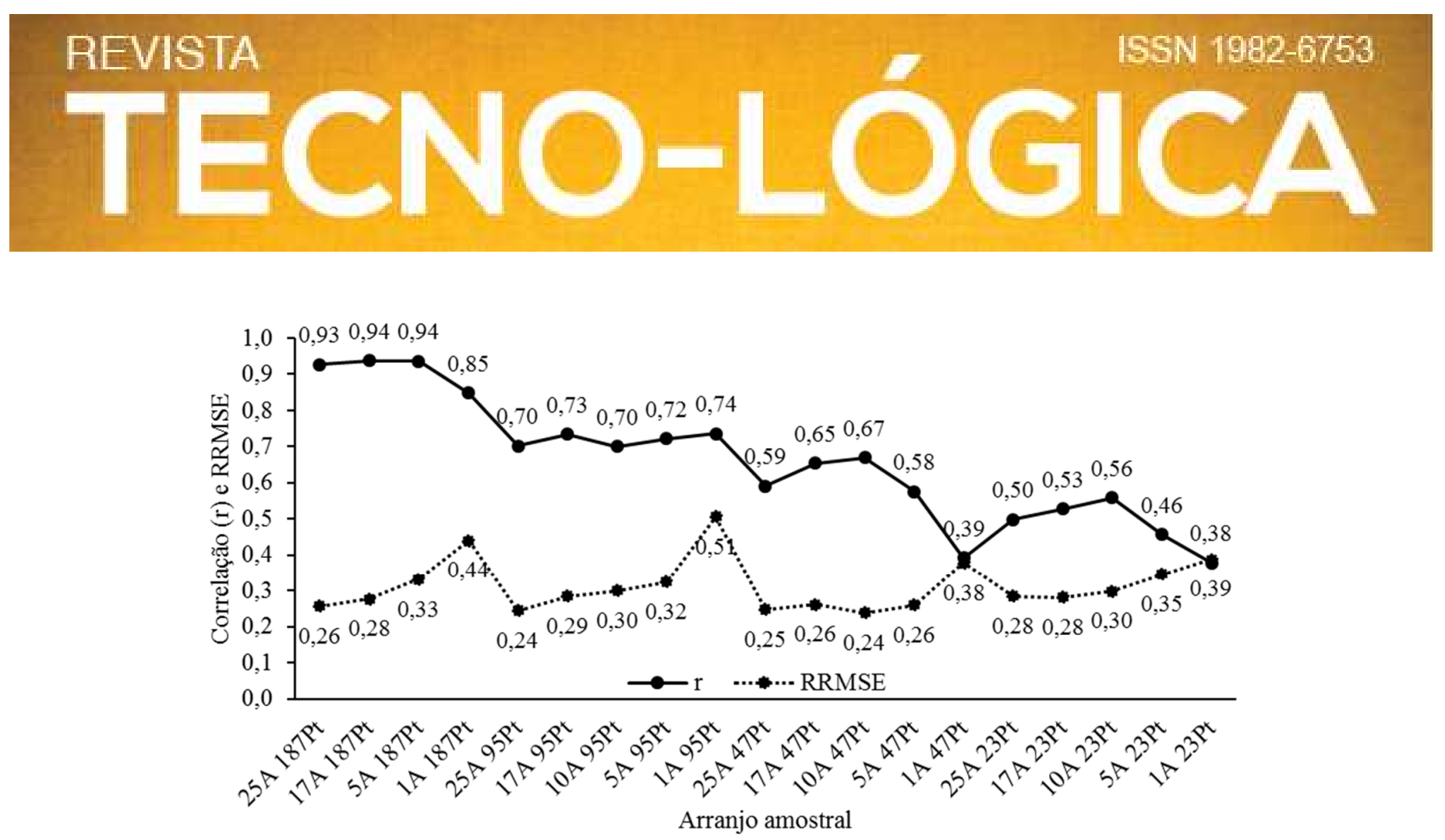

Figura 2. Correlação (r) e valores de RRMSE do mapa de variabilidade com melhor ajuste do variograma e validação cruzada para o K (10A 187Pt) comparado com os mapas dos restantes arranjos amostrais.

Os melhores ajustes dos modelos teóricos também foram obtidos nos arranjos amostrais com a malha mais densa utilizada. Com o distanciamento das amostras, ficou evidente o aumento da SQR e a redução dos coeficientes de determinação do modelo ajustado indicando perda de qualidade do ajuste nas malhas menos densas. Com exceção da malha amostral com 187 pontos, nos demais arranjos formados por uma amostra simples, foi observado efeito pepita puro e, no arranjo com 187 pontos, a utilização de uma amostra simples causou deterioração do ajuste do modelo comparado ao restante dos arranjos amostrais com 187 pontos e mais de uma amostra simples por ponto. O IDE para V\% também foi classificado como moderado a forte (50 a $100 \%$ ), sem aumento do efeito pepita conforme se distanciam os pontos amostrais.
Pela validação cruzada dos dados, observou-se não haver diferenças marcantes entre a RQEM e o EMP calculados (Tabela 7), entretanto, os coeficientes de regressão diminuíram com a utilização de arranjos amostrais com 95 pontos ou menos. O coeficiente de regressão também foi prejudicado quando se utilizou uma amostra simples, mesmo com 187 pontos amostrais. Para o V\%, não foram observadas melhorias na precisão da krigagem, quando se utilizou mais do que 5 amostras simples para formar a amostra composta.

TECNO-LÓGICA, Santa Cruz do Sul, v. 25, n. 1, p. 145-172, jul./dez. 2021 A matéria publicada nesse periódico é licenciada sob forma de uma http://creativecommons.org/licenses/by/4.0/ 


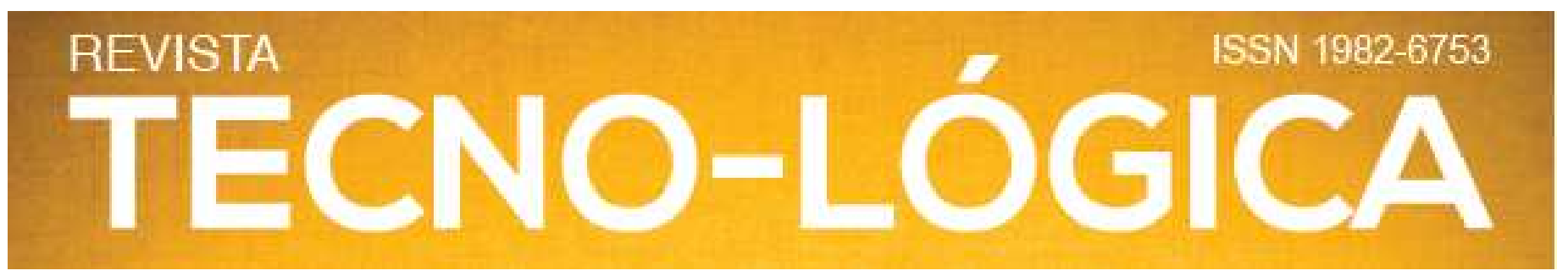

Tabela 6. Parâmetros dos modelos teóricos dos semivariogramas ajustados para a saturação por bases no solo (V) nos diferentes arranjos amostrais utilizados

\begin{tabular}{|c|c|c|c|c|c|c|c|c|}
\hline Arranjo & Pontos excluídos & Modelo & Alcance (m) & $\mathrm{C}_{0}+\mathrm{C}^{(3)}$ & $\mathrm{C}_{0}^{(4)}$ & IDE $^{(5)}$ & $\mathbf{S Q R}^{(\mathbf{6})}$ & $\mathbf{R}^{2(7)}$ \\
\hline $25 \mathrm{~A}^{(1)} 187 \mathrm{Pt}^{(2)}$ & 4 & $\mathrm{ESF}^{(8)}$ & 264 & 33,89 & 16,49 & 50 & 3,30 & 0,97 \\
\hline 17 A $187 \mathrm{Pt}$ & 4 & ESF & 276 & 32,15 & 15,63 & 51 & 2,44 & 0,98 \\
\hline $10 \mathrm{~A} 187 \mathrm{Pt}$ & 3 & ESF & 286 & 40,03 & 20,01 & 50 & 4,46 & 0,97 \\
\hline $5 \mathrm{~A} 187 \mathrm{Pt}$ & 4 & ESF & 372 & 40,83 & 18,48 & 54 & 7,68 & 0,97 \\
\hline $1 \mathrm{~A} 187 \mathrm{Pt}$ & 3 & ESF & 103 & 43,73 & 0,50 & 98 & 34,7 & 0,40 \\
\hline 25 A $95 \mathrm{Pt}$ & 3 & ESF & 127 & 30,32 & 0,01 & 100 & 20,7 & 0,40 \\
\hline 17 A $95 \mathrm{Pt}$ & 3 & ESF & 256 & 26,05 & 11,98 & 54 & 11,0 & 0,80 \\
\hline $10 \mathrm{~A} 95 \mathrm{Pt}$ & 0 & ESF & 207 & 39,86 & 1,30 & 96 & 25,4 & 0,88 \\
\hline 5 A $95 \mathrm{Pt}$ & 1 & ESF & 589 & 44,21 & 20,85 & 52 & 30,6 & 0,92 \\
\hline $1 \mathrm{~A} 95 \mathrm{Pt}$ & 1 & $\mathrm{EPP}^{(9)}$ & - & 48,72 & 48,72 & - & - & - \\
\hline $25 \mathrm{~A} 47 \mathrm{Pt}$ & 2 & ESF & 221 & 27,76 & 1,21 & 95 & 21,2 & 0,43 \\
\hline $17 \mathrm{~A} 47 \mathrm{Pt}$ & 1 & EPP & - & 34,69 & 34,69 & - & - & - \\
\hline $10 \mathrm{~A} 47 \mathrm{Pt}$ & 0 & ESF & 177 & 42,23 & 2,10 & 95 & 2,75 & 0,58 \\
\hline $5 \mathrm{~A} 47 \mathrm{Pt}$ & 0 & ESF & 205 & 40,37 & 0,10 & 99 & 33,6 & 0,38 \\
\hline $1 \mathrm{~A} 47 \mathrm{Pt}$ & 0 & EPP & - & 39,66 & 39,66 & - & - & - \\
\hline $25 \mathrm{~A} 23 \mathrm{Pt}$ & 2 & ESF & 350 & 25,24 & 1,30 & 94 & 31,6 & 0,42 \\
\hline 17 A $23 \mathrm{Pt}$ & 2 & $\operatorname{EXP}^{(10)}$ & 387 & 23,59 & 3,78 & 84 & 13,4 & 0,84 \\
\hline 10 A $23 \mathrm{Pt}$ & 0 & EPP & - & 36,98 & 36,98 & - & - & - \\
\hline $5 \mathrm{~A} 23 \mathrm{Pt}$ & 0 & ESF & 305 & 37,19 & 1,10 & 97 & 13,8 & 0,62 \\
\hline $1 \mathrm{~A} 23 \mathrm{Pt}$ & 0 & EPP & - & 32,57 & 32,57 & - & - & - \\
\hline
\end{tabular}

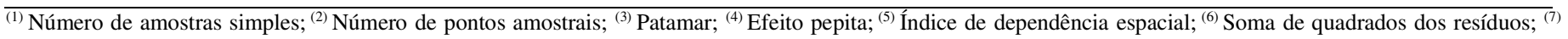

Coeficiente de determinação; ${ }^{(8)}$ Esférico; ${ }^{(9)}$ Efeito pepita puro; ${ }^{(10)}$ Exponencial.

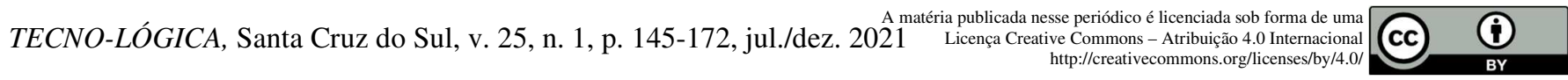




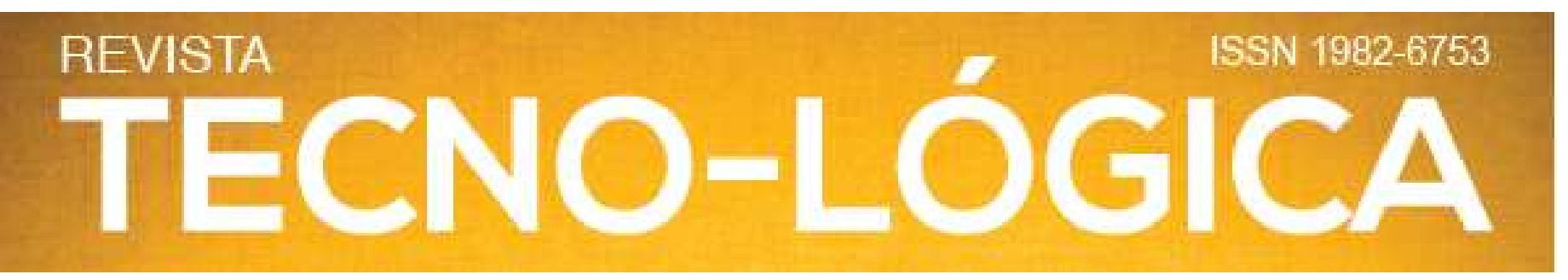

Tabela 7. Validação cruzada dos modelos teóricos dos semivariogramas ajustados para a saturação por bases (V) do solo nos diferentes arranjos amostrais utilizados

\begin{tabular}{|c|c|c|c|c|c|c|}
\hline Arranjo & Coeficiente de regressão & Intercepto (Y) & Erro padrão (SE) & $\mathbf{R}^{2(3)}$ & RQEM $^{(4)}$ & $\mathbf{E M P}^{(5)}$ \\
\hline $25 \mathrm{~A}^{(1)} 187 \mathrm{Pt}^{(2)}$ & 0,843 & 10,83 & 0,141 & 0,165 & 5,18 & 6,12 \\
\hline 17 A $187 \mathrm{Pt}$ & 0,872 & 8,56 & 0,140 & 0,176 & 4,95 & 6,10 \\
\hline $10 \mathrm{~A} 187 \mathrm{Pt}$ & 0,872 & 8,53 & 0,135 & 0,187 & 5,50 & 6,53 \\
\hline $5 \mathrm{~A} 187 \mathrm{Pt}$ & 0,984 & 1,10 & 0,102 & 0,339 & 5,18 & 6,03 \\
\hline $1 \mathrm{~A} 187 \mathrm{Pt}$ & 0,488 & 34,34 & 0,141 & 0,062 & 6,62 & 7,98 \\
\hline $25 \mathrm{~A} 95 \mathrm{Pt}$ & 0,315 & 46,75 & 0,149 & 0,047 & 5,91 & 7,30 \\
\hline 17 A $95 \mathrm{Pt}$ & 0,558 & 29,50 & 0,210 & 0,073 & 4,98 & 6,19 \\
\hline $10 \mathrm{~A} 95 \mathrm{Pt}$ & 0,482 & 34,10 & 0,147 & 0,103 & 6,30 & 7,81 \\
\hline $5 \mathrm{~A} 95 \mathrm{Pt}$ & 1,010 & $-0,61$ & 0,148 & 0,336 & 5,36 & 6,26 \\
\hline $25 \mathrm{~A} 47 \mathrm{Pt}$ & 0,692 & 21,09 & 0,288 & 0,119 & 4,85 & 6,05 \\
\hline $10 \mathrm{~A} 47 \mathrm{Pt}$ & 0,210 & 51,64 & 0,248 & 0,016 & 6,91 & 9,40 \\
\hline $5 \mathrm{~A} 47 \mathrm{Pt}$ & 0,619 & 25,54 & 0,249 & 0,121 & 6,09 & 7,42 \\
\hline $25 \mathrm{~A} 23 \mathrm{Pt}$ & 0,592 & 28,12 & 0,380 & 0,108 & 3,91 & 4,98 \\
\hline $17 \mathrm{~A} 23 \mathrm{Pt}$ & 0,878 & 8,35 & 0,340 & 0,260 & 3,26 & 3,96 \\
\hline $5 \mathrm{~A} 23 \mathrm{Pt}$ & 0,761 & 16,28 & 0,340 & 0,193 & 5,59 & 6,94 \\
\hline
\end{tabular}

${ }^{(1)}$ Número de amostras simples; ${ }^{(2)}$ Número de pontos amostrais; ${ }^{(3)}$ Coeficiente de determinação; ${ }^{(4)}$ Raiz quadrada do erro médio; ${ }^{(5)}$ Erro médio percentual.

Os mapas da saturação por bases apresentaram correlações classificadas como forte no arranjo amostral mais denso, com exceção do arranjo que possui uma amostra simples.

Diferentemente do $\mathrm{P}$ e $\mathrm{K}$ avaliados anteriormente, os arranjos contendo 5 amostras simples por ponto apresentaram correlações classificadas como muito forte para a densidade de 95 pontos e como forte paras as densidades de 47 e 23 pontos (Figura 3).

Provavelmente, este fato decorreu da utilização do mesmo conjunto de dados (análises de solo), visto que o melhor ajuste dos semivariogramas se deu com o arranjo composto por
5 amostras simples, combinado com 187 pontos amostrais (5A 187Pt). Em todos os arranjos obteve-se valores de RRMSE semelhantes. Supõe-se que exista grande variabilidade horizontal dos teores de bases trocáveis no solo, resultando em grande variação dos teores de $\mathrm{Ca}$ e $\mathrm{Mg}$ no solo, e que, conforme se varia o número de amostras simples coletadas, ocorra alteração do resultado das análises. Essas grandes variações podem ser resultantes de calagens e gessagens sucessivas e irregulares. 

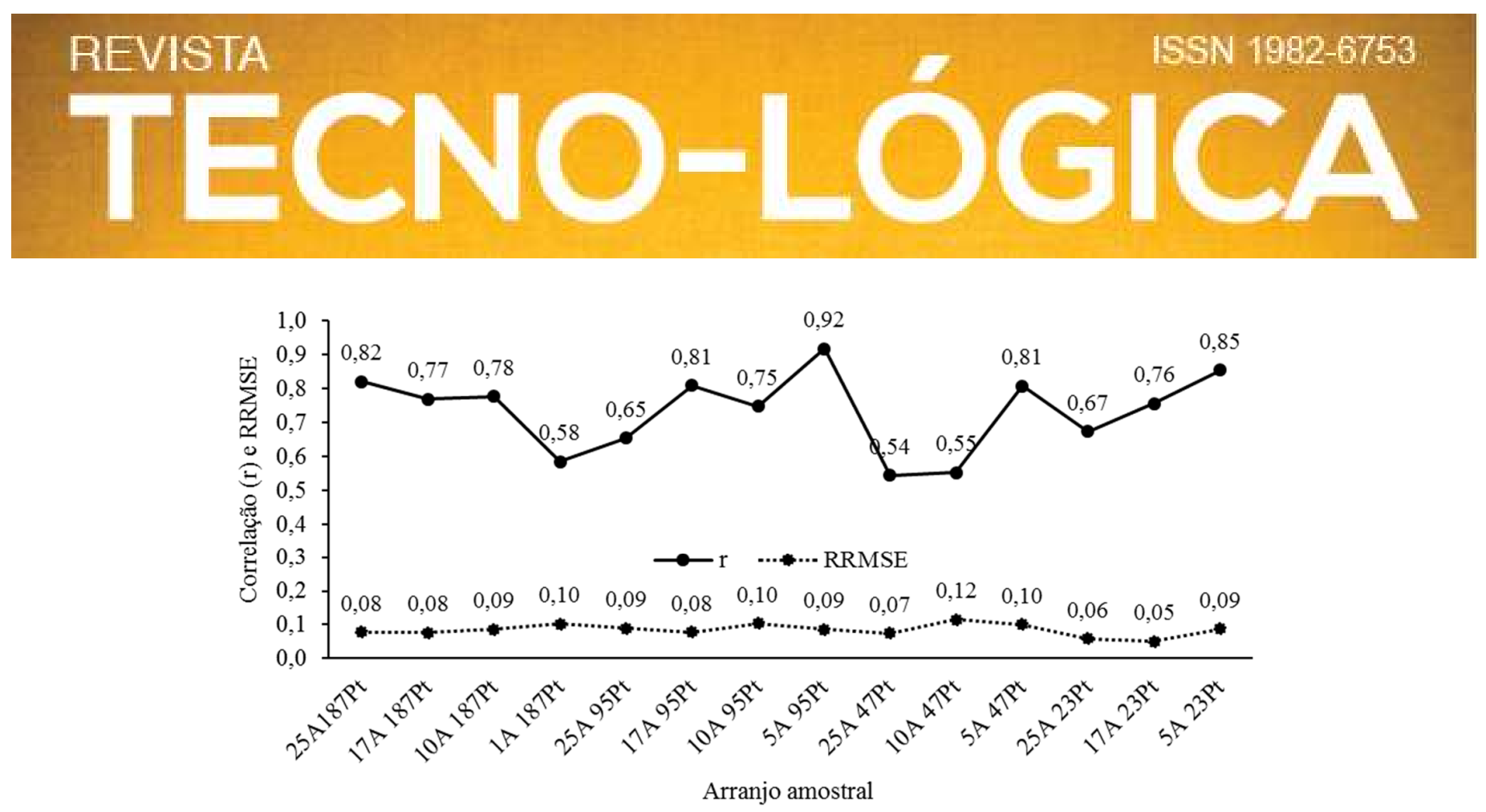

Figura 3. Correlação (r) e valores de RRMSE do mapa de variabilidade com melhor ajuste do variograma e validação cruzada para a saturação por bases (5A 187Pt), comparado com os mapas dos restantes arranjos amostrais.

A dependência espacial do $\mathrm{pH}$ do solo, quando existente, foi melhor ajustada pelo modelo esférico (Tabela 8). Para este atributo do solo, foram observados bons ajustes do semivariograma nos arranjos formados com 187 e 95 pontos amostrais, exceto os formados por uma amostra simples por ponto. O alcance da dependência espacial apresentou menor amplitude de variação, comparado aos atributos anteriores, variando de $147 \mathrm{~m}$ (1 amostra simples com 187 pontos) a $398 \mathrm{~m}$ (10 amostras simples com 47 pontos). Os valores de alcance podem influenciar na qualidade das estimativas, uma vez que ele determina o número de valores usados na interpolação, assim, estimativas feitas com interpolação por krigagem ordinária utilizando valores de alcances maiores tendem a ser mais confiáveis, apresentando mapas que representem melhor a realidade [23].

Zanão Júnior et al. [24] destacam que a variabilidade espacial dos nutrientes pode não ser igual entre eles, pois alguns nutrientes necessitam de números maiores de amostras que outros para que se possa conhecer o seu comportamento em relação a uma determinada área. Isso acontece porque as variações nos teores dos elementos do solo não são uniformes para todos [25]. O IDE para esse atributo também foi classificado como de moderado a forte, sem evidências de aumento do efeito pepita com o distanciamento dos pontos, ou com a redução do número de amostras simples. 


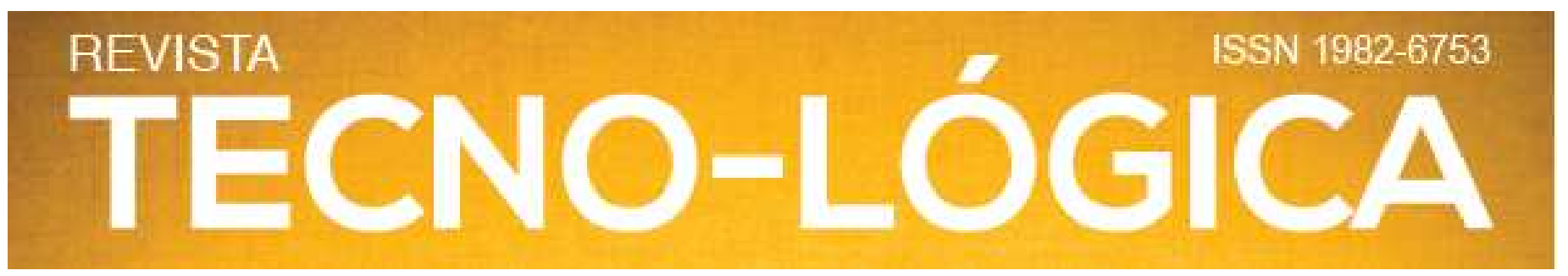

Tal semelhança pode ser indicativa de que o uso de homogênea ao longo dos 30 anos de cultivo do talhão, que não malhas mais densas não melhore os parâmetros da validação, obedecem a padrões de formação de solo e relevo, e, dessa principalmente o $\mathrm{R}^{2}$. Os baixos valores do $\mathrm{R}^{2}$ da validação forma, prejudicam o ajuste do $\mathrm{R}^{2}$ da validação cruzada, mesmo cruzada podem ser explicados por fatores extrínsecos do solo tais em malhas mais intensas. como adições de corretivos e fertilizantes de forma não

Tabela 8. Parâmetros dos modelos teóricos dos semivariogramas ajustados para o $\mathrm{pH}$ do solo $\left(\mathrm{CaCl}_{2}\right)$ nos diferentes arranjos amostrais utilizados

\begin{tabular}{|c|c|c|c|c|c|c|c|c|}
\hline Arranjo & Pontos excluídos & Modelo & Alcance (m) & $\mathrm{C}_{0}+\mathrm{C}^{(3)}$ & $\mathrm{C}_{0}{ }^{(4)}$ & IDE $^{(5)}$ & $\mathbf{S Q R}^{(6)}$ & $\mathbf{R}^{2(7)}$ \\
\hline $25 \mathrm{~A}^{(1)} 187 \mathrm{Pt}^{(2)}$ & 2 & $\mathrm{ESF}^{(8)}$ & 240 & 0,0536 & 0,0087 & 83 & $3,2 \times \cdot 10^{-5}$ & 0,93 \\
\hline 17 A $187 \mathrm{Pt}$ & 0 & ESF & 241 & 0,0564 & 0,0110 & 80 & $5,9 x \cdot 10^{-5}$ & 0,88 \\
\hline 10 A $187 \mathrm{Pt}$ & 1 & ESF & 361 & 0,0584 & 0,0208 & 64 & $4,4 x \cdot 10^{-5}$ & 0,93 \\
\hline $5 \mathrm{~A} 187 \mathrm{Pt}$ & 0 & ESF & 254 & 0,0650 & 0,0158 & 75 & $4,1 \times \cdot 10^{-5}$ & 0,93 \\
\hline $1 \mathrm{~A} 187 \mathrm{Pt}$ & 0 & ESF & 147 & 0,1032 & 0,0051 & 95 & $2,0 x \cdot 10^{-4}$ & 0,69 \\
\hline 25 A $95 \mathrm{Pt}$ & 0 & ESF & 236 & 0,0510 & 0,0001 & 99 & $2,3 x \cdot 10^{-5}$ & 0,97 \\
\hline 17 A $95 \mathrm{Pt}$ & 0 & ESF & 251 & 0,0501 & 0,0001 & 99 & $3,2 x \cdot 10^{-5}$ & 0,97 \\
\hline 10 A $95 \mathrm{Pt}$ & 0 & ESF & 282 & 0,0546 & 0,0004 & 99 & $6,1 \times \cdot 10^{-5}$ & 0,95 \\
\hline $5 \mathrm{~A} 95 \mathrm{Pt}$ & 0 & ESF & 280 & 0,0598 & 0,0001 & 99 & $1,3 x \cdot 10^{-4}$ & 0,92 \\
\hline $1 \mathrm{~A} 95 \mathrm{Pt}$ & 0 & ESF & 169 & 0,0952 & 0,0036 & 96 & $1,1 \times \cdot 10^{-4}$ & 0,89 \\
\hline $25 \mathrm{~A} 47 \mathrm{Pt}$ & 0 & ESF & 202 & 0,0483 & 0,0028 & 94 & $1,0 x \cdot 10^{-5}$ & 0,69 \\
\hline $17 \mathrm{~A} 47 \mathrm{Pt}$ & 0 & ESF & 205 & 0,0515 & 0,0019 & 96 & $2,8 x \cdot 10^{-5}$ & 0,52 \\
\hline $10 \mathrm{~A} 47 \mathrm{Pt}$ & 0 & ESF & 398 & 0,0566 & 0,0282 & 50 & $2,5 x \cdot 10^{-5}$ & 0,83 \\
\hline $5 \mathrm{~A} 47 \mathrm{Pt}$ & 0 & ESF & 200 & 0,0620 & 0,0051 & 91 & $1,6 x \cdot 10^{-4}$ & 0,17 \\
\hline $1 \mathrm{~A} 47 \mathrm{Pt}$ & 0 & $\mathrm{EPP}^{(9)}$ & - & 0,0785 & 0,0785 & - & - & - \\
\hline $25 \mathrm{~A} 23 \mathrm{Pt}$ & 0 & ESF & 305 & 0,0452 & 0,0001 & 99 & $2,6 x \cdot 10^{-4}$ & 0,31 \\
\hline 17 A $23 \mathrm{Pt}$ & 0 & ESF & 347 & 0,0537 & 0,0001 & 99 & $1,8 x \cdot 10^{-4}$ & 0,54 \\
\hline 10 A $23 \mathrm{Pt}$ & 0 & ESF & 293 & 0,0475 & 0,0006 & 98 & $3,6 x \cdot 10^{-4}$ & 0,14 \\
\hline $5 \mathrm{~A} 23 \mathrm{Pt}$ & 0 & ESF & 334 & 0,0590 & 0,0001 & 99 & $1,3 \times \cdot 10^{-3}$ & 0,17 \\
\hline $1 \mathrm{~A} 23 \mathrm{Pt}$ & 0 & EPP & - & 0,0546 & 0,0546 & - & - & - \\
\hline
\end{tabular}

${ }^{(1)}$ Número de amostras simples; ${ }^{(2)}$ Número de pontos amostrais; ${ }^{(3)}$ Patamar; ${ }^{(4)}$ Efeito pepita; ${ }^{(5)}$ Índice de dependência espacial; ${ }^{(6)}$ Soma de quadrados dos resíduos; ${ }^{(7)}$ Coeficiente de determinação; ${ }^{(8)}$ Esférico; ${ }^{(9)}$ Efeito pepita puro.

Pela validação cruzada (Tabela 9), verificou-se que os arranjos formados por 187 e 95 pontos amostrais com pelo menos 5 amostras simples apresentaram os parâmetros da validação muito semelhantes.

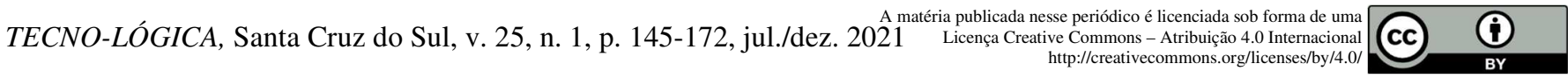
161 


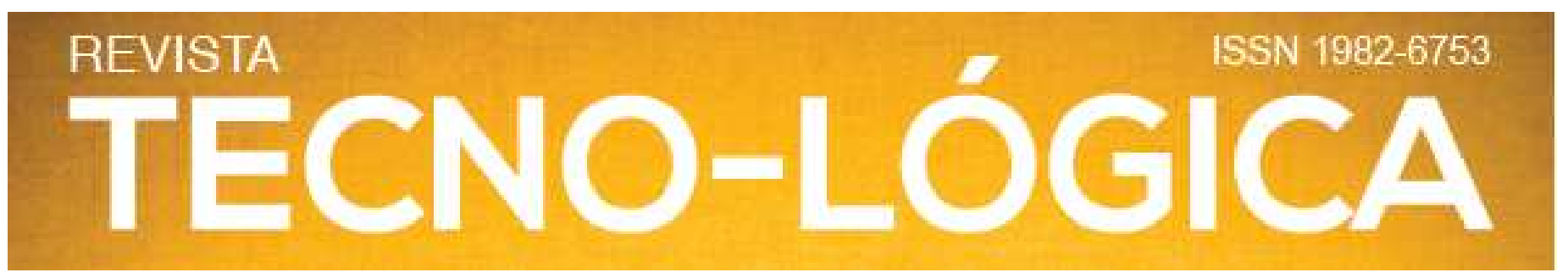

Tabela 9. Validação cruzada dos modelos teóricos dos semivariogramas ajustados para o pH do solo $\left(\mathrm{CaCl}_{2}\right)$ nos diferentes arranjos amostrais utilizados

\begin{tabular}{|c|c|c|c|c|c|c|}
\hline Arranjo & Coeficiente de regressão & Intercepto (Y) & Erro padrão (SE) & $\mathbf{R}^{2(3)}$ & RQEM $^{(4)}$ & $\mathbf{E M P}^{(5)}$ \\
\hline $25 \mathrm{~A}^{(1)} 187 \mathrm{Pt}^{(2)}$ & 0,832 & 0,88 & 0,089 & 0,324 & 0,184 & 2,89 \\
\hline 17 A $187 \mathrm{Pt}$ & 0,862 & 0,72 & 0,092 & 0,324 & 0,187 & 2,87 \\
\hline $10 \mathrm{~A} 187 \mathrm{Pt}$ & 1,017 & $-0,08$ & 0,104 & 0,341 & 0,182 & 2,82 \\
\hline $5 \mathrm{~A} 187 \mathrm{Pt}$ & 0,871 & 0,67 & 0,098 & 0,297 & 0,206 & 3,07 \\
\hline $1 \mathrm{~A} 187 \mathrm{Pt}$ & 0,642 & 1,87 & 0,107 & 0,163 & 0,295 & 4,60 \\
\hline $25 \mathrm{~A} 95 \mathrm{Pt}$ & 0,804 & 1,03 & 0,125 & 0,308 & 0,185 & 2,87 \\
\hline 17 A $95 \mathrm{Pt}$ & 0,853 & 0,77 & 0,118 & 0,360 & 0,176 & 2,77 \\
\hline $10 \mathrm{~A} 95 \mathrm{Pt}$ & 0,854 & 0,76 & 0,119 & 0,356 & 0,178 & 2,82 \\
\hline $5 \mathrm{~A} 95 \mathrm{Pt}$ & 0,880 & 0,63 & 0,120 & 0,365 & 0,188 & 2,94 \\
\hline $1 \mathrm{~A} 95 \mathrm{Pt}$ & 0,394 & 3,18 & 0,172 & 0,053 & 0,312 & 4,76 \\
\hline $25 \mathrm{~A} 47 \mathrm{Pt}$ & 0,244 & 3,93 & 0,307 & 0,014 & 0,223 & 3,58 \\
\hline $17 \mathrm{~A} 47 \mathrm{Pt}$ & 0,428 & 2,98 & 0,277 & 0,050 & 0,225 & 3,49 \\
\hline $10 \mathrm{~A} 47 \mathrm{Pt}$ & 0,648 & 1,83 & 0,325 & 0,081 & 0,214 & 3,34 \\
\hline $5 \mathrm{~A} 47 \mathrm{Pt}$ & 0,409 & 3,06 & 0,277 & 0,046 & 0,242 & 3,74 \\
\hline $25 \mathrm{~A} 23 \mathrm{Pt}$ & 0,183 & 4,24 & 0,496 & 0,006 & 0,230 & 3,95 \\
\hline 17 A $23 \mathrm{Pt}$ & 0,490 & 2,64 & 0,399 & 0,067 & 0,234 & 3,97 \\
\hline $10 \mathrm{~A} 23 \mathrm{Pt}$ & 0,354 & 3,35 & 0,399 & 0,036 & 0,232 & 3,81 \\
\hline 5 A $23 \mathrm{Pt}$ & 0,850 & 0,78 & 0,364 & 0,206 & 0,223 & 3,37 \\
\hline
\end{tabular}

${ }^{(1)}$ Número de amostras simples; ${ }^{(2)}$ Número de pontos amostrais; ${ }^{(3)}$ Coeficiente de determinação; ${ }^{(4)}$ Raiz quadrada do erro médio; ${ }^{(5)}$ Erro médio percentual.

Para os mapas de pH da solução do solo (Figura 4), foram observadas correlações muito fortes e fortes para os arranjos contendo 187 e 95 pontos, respectivamente, exceto os combinados com apenas uma amostra simples por ponto. Nos arranjos formados por 47 pontos, exceto o formado por 1 amostra simples e nos arranjos formados por 23 pontos com 10 e 5 amostras simples, também foram observadas fortes correlações, provavelmente pela maior similaridade do conjunto de dados nos arranjos compostos por 5 amostras simples, quando este comparado aos formados por 17 e 25 , visto que o mapa com melhor qualidade foi obtido pelo arranjo formado por 187 pontos e 10 amostras simples (mapa padrão). Dessa forma, explica-se as melhores correlações obtidas em todos os arranjos formados por 10 amostras simples, pois o conjunto de dados é o mesmo do mapa de comparação. A acurácia de todos os mapas (RRMSE) foram muito semelhantes, contudo, nota-se um aumento mais pronunciado do RRMSE nos arranjos contendo apenas uma amostra simples.

TECNO-LÓGICA, Santa Cruz do Sul, v $25, \mathrm{n}, 1, \mathrm{p}, 145-172, \mathrm{jul} / \mathrm{dez} .2021$ A matéria publicada nesse periódico é licenciada sob forma de uma 

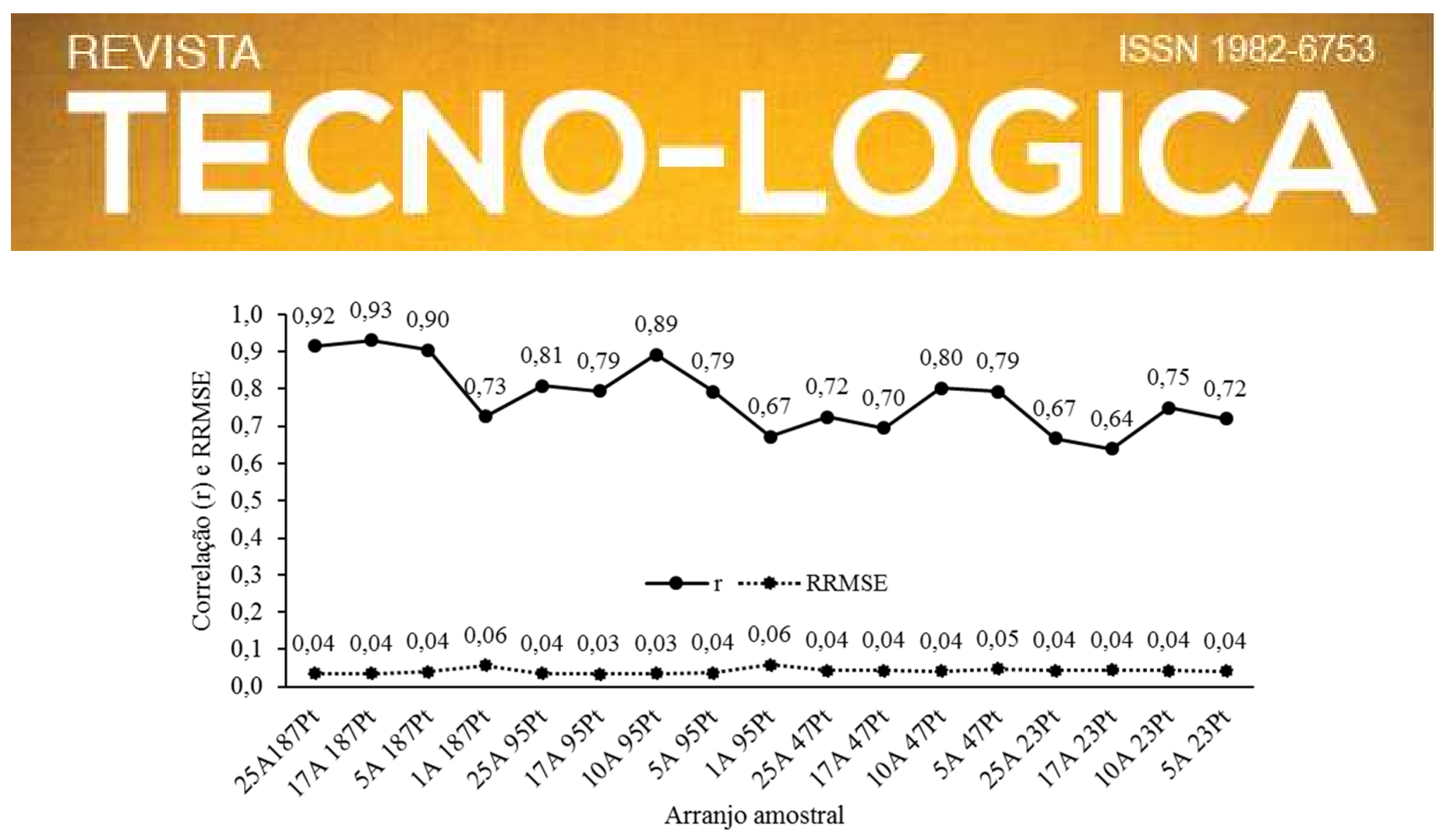

Figura 4. Correlação (r) e valores de RRMSE do mapa de variabilidade com melhor ajuste do variograma e validação cruzada para o pH do solo (10A 187Pt) comparado com os mapas dos restantes arranjos amostrais.

Para a M.O. (Tabela 10), os semivariogramas apresentaram estruturas semelhantes nos arranjos formados com 187, 95 e 47 pontos amostrados. Para esse atributo, observa-se menor perda de qualidade do ajuste do modelo teórico nos arranjos formados com uma amostra simples, mas a deterioração dos parâmetros do modelo teórico ainda é evidente quando se utiliza tal amostra combinada com 95 pontos ou menos. Apesar disso, esse atributo apresenta moderada a forte dependência espacial.

Os parâmetros da validação cruzada (Tabela 11) confirmam a perda de precisão dos arranjos formados com uma amostra simples por ponto e também nos semivariogramas que utilizaram menos de 95 pontos amostrais, não apresentando diferença entre as malhas contendo 187 e 95 pontos amostrais.

Os mapas dos teores de M.O. apresentaram correlações de fortes a muito fortes em todos os arranjos avaliados, exceto ao composto por 23 pontos combinado com cinco amostras simples (Figura 5). Os valores do RRMSE foram semelhantes em todos os arranjos amostrais 


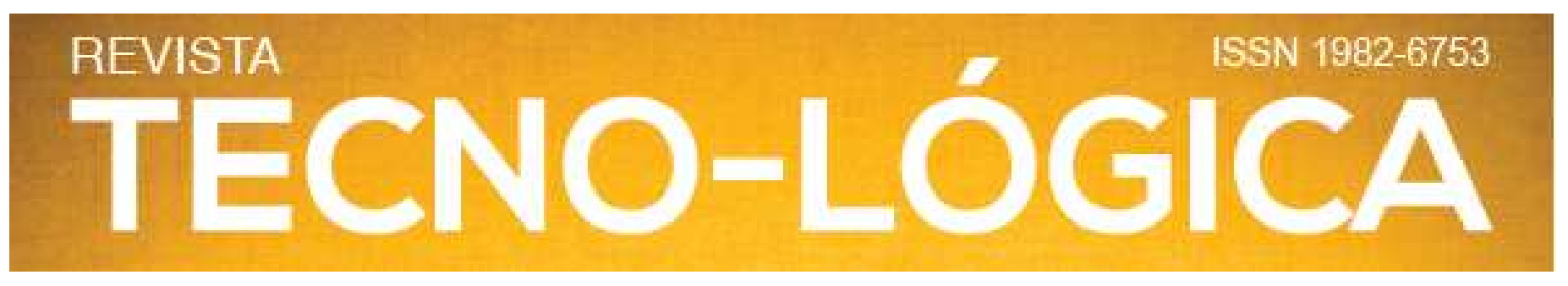

Tabela 10. Parâmetros dos modelos teóricos dos semivariogramas ajustados para a MO nos diferentes arranjos amostrais utilizados

\begin{tabular}{|c|c|c|c|c|c|c|c|c|}
\hline Arranjo & Pontos excluídos & Modelo & Alcance (m) & $\mathrm{C}_{0}+\mathrm{C}^{(3)}$ & $\mathrm{C}_{0}{ }^{(4)}$ & IDE $^{(5)}$ & $\mathbf{S Q R}^{(6)}$ & $\mathbf{R}^{2(7)}$ \\
\hline $25 \mathrm{~A}^{(1)} 187 \mathrm{Pt}^{(2)}$ & 2 & $\mathrm{GAU}^{(8)}$ & 495 & 31,96 & 10,05 & 68 & 1,1 & 0,99 \\
\hline $17 \mathrm{~A} 187 \mathrm{Pt}$ & 2 & GAU & 503 & 28,49 & 9,14 & 67 & 3,2 & 0,99 \\
\hline $10 \mathrm{~A} 187 \mathrm{Pt}$ & 2 & GAU & 509 & 24,46 & 8,29 & 66 & 0,4 & 0,99 \\
\hline 5 A $187 \mathrm{Pt}$ & 0 & GAU & 521 & 30,13 & 10,28 & 65 & 1,7 & 0,99 \\
\hline $1 \mathrm{~A} 187 \mathrm{Pt}$ & 1 & GAU & 426 & 28,57 & 13,62 & 52 & 2,9 & 0,99 \\
\hline 25 A $95 \mathrm{Pt}$ & 0 & GAU & 517 & 36,28 & 9,96 & 72 & 5,5 & 0,99 \\
\hline 17 A $95 \mathrm{Pt}$ & 0 & GAU & 572 & 27,95 & 7,04 & 74 & 6,1 & 0,98 \\
\hline 10 A $95 \mathrm{Pt}$ & 2 & GAU & 598 & 22,80 & 7,92 & 65 & 3,5 & 0,98 \\
\hline 5 A $95 \mathrm{Pt}$ & 1 & GAU & 502 & 25,47 & 8,50 & 66 & 3,8 & 0,99 \\
\hline $1 \mathrm{~A} 95 \mathrm{Pt}$ & 2 & GAU & 665 & 32,29 & 16,14 & 50 & 15,8 & 0,89 \\
\hline $25 \mathrm{~A} 47 \mathrm{Pt}$ & 0 & GAU & 523 & 24,56 & 12,25 & 50 & 5,0 & 0,95 \\
\hline $17 \mathrm{~A} 47 \mathrm{Pt}$ & 0 & GAU & 756 & 23,62 & 8,01 & 66 & 1,2 & 0,97 \\
\hline $10 \mathrm{~A} 47 \mathrm{Pt}$ & 0 & GAU & 457 & 12,39 & 6,17 & 50 & 3,8 & 0,89 \\
\hline $5 \mathrm{~A} 47 \mathrm{Pt}$ & 0 & GAU & 315 & 11,89 & 5,94 & 50 & 1,2 & 0,95 \\
\hline $1 \mathrm{~A} 47 \mathrm{Pt}$ & 0 & $\operatorname{ESF}^{(9)}$ & 323 & 13,83 & 0,71 & 94 & 17,0 & 0,55 \\
\hline 25 A $23 \mathrm{Pt}$ & 0 & $\operatorname{EXP}^{(10)}$ & 1313 & 29,95 & 12,54 & 58 & 5,4 & 0,84 \\
\hline 17 A $23 \mathrm{Pt}$ & 0 & EXP & 752 & 16,42 & 6,31 & 61 & 1,5 & 0,91 \\
\hline $10 \mathrm{~A} 23 \mathrm{Pt}$ & 0 & EXP & 3110 & 20,00 & 5,33 & 73 & 6,7 & 0,47 \\
\hline 5 A $23 \mathrm{Pt}$ & 0 & EXP & 3110 & 19,74 & 7,36 & 67 & 11,6 & 0,39 \\
\hline $1 \mathrm{~A} 23 \mathrm{Pt}$ & 0 & $\mathrm{EPP}^{(11)}$ & - & 10,41 & 10,41 & - & - & - \\
\hline
\end{tabular}

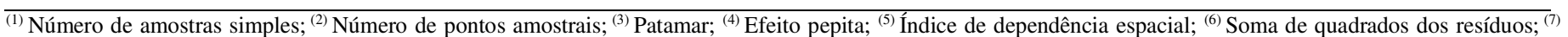

Coeficiente de determinação; ${ }^{(8)}$ Gaussiano; ${ }^{(9)}$ Esférico; ${ }^{(10)}$ Exponencial; ${ }^{(11)}$ Efeito pepita puro

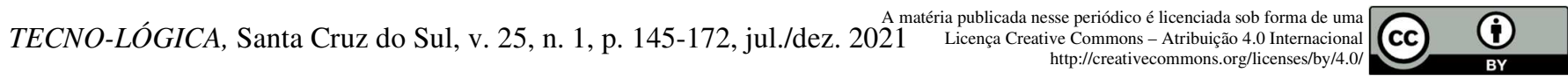




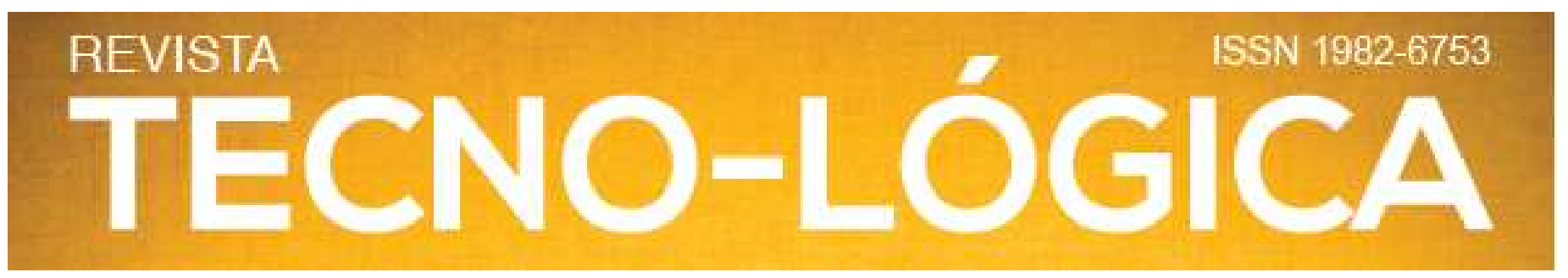

Tabela 11. Validação cruzada dos modelos teóricos de semivariância ajustados para a MO nos diferentes arranjos amostrais utilizados

\begin{tabular}{|c|c|c|c|c|c|c|}
\hline Arranjo & Coeficiente de regressão & Intercepto (Y) & Erro padrão (SE) & $\mathbf{R}^{2(3)}$ & RQEM $^{(4)}$ & $\mathbf{E M P}^{(5)}$ \\
\hline $25 \mathrm{~A}^{(1)} 187 \mathrm{Pt}^{(2)}$ & 1,015 & $-0,44$ & 0,079 & 0,477 & 3,359 & 9,08 \\
\hline 17 A $187 \mathrm{Pt}$ & 1,006 & $-0,16$ & 0,083 & 0,466 & 3,237 & 8,99 \\
\hline $10 \mathrm{~A} 187 \mathrm{Pt}$ & 1,034 & $-0,98$ & 0,079 & 0,483 & 2,947 & 7,93 \\
\hline $5 \mathrm{~A} 187 \mathrm{Pt}$ & 1,018 & $-0,49$ & 0,086 & 0,429 & 3,429 & 9,12 \\
\hline $1 \mathrm{~A} 187 \mathrm{Pt}$ & 0,975 & 0,73 & 0,101 & 0,335 & 3,883 & 11,43 \\
\hline $25 \mathrm{~A} 95 \mathrm{Pt}$ & 1,059 & $-1,79$ & 0,113 & 0,484 & 3,454 & 9,72 \\
\hline 17 A $95 \mathrm{Pt}$ & 1,111 & $-3,27$ & 0,108 & 0,533 & 2,850 & 7,84 \\
\hline $10 \mathrm{~A} 95 \mathrm{Pt}$ & 1,082 & $-2,40$ & 0,128 & 0,439 & 2,805 & 7,94 \\
\hline $5 \mathrm{~A} 95 \mathrm{Pt}$ & 1,021 & $-0,61$ & 0,138 & 0,375 & 3,259 & 8,95 \\
\hline $1 \mathrm{~A} 95 \mathrm{Pt}$ & 1,074 & $-2,05$ & 0,189 & 0,261 & 3,995 & 11,58 \\
\hline $25 \mathrm{~A} 47 \mathrm{Pt}$ & 0,862 & 4,03 & 0,314 & 0,143 & 3,857 & 11,43 \\
\hline $17 \mathrm{~A} 47 \mathrm{Pt}$ & 1,017 & $-3,11$ & 0,290 & 0,245 & 3,088 & 8,77 \\
\hline $10 \mathrm{~A} 47 \mathrm{Pt}$ & 0,942 & 1,70 & 0,252 & 0,236 & 2,739 & 7,86 \\
\hline $5 \mathrm{~A} 47 \mathrm{Pt}$ & 0,638 & 10,23 & 0,281 & 0,103 & 3,108 & 8,68 \\
\hline $1 \mathrm{~A} 47 \mathrm{Pt}$ & 0,557 & 12,59 & 0,200 & 0,147 & 3,481 & 10,09 \\
\hline $25 \mathrm{~A} 23 \mathrm{Pt}$ & 0,233 & 21,54 & 0,809 & 0,004 & 4,447 & 13,48 \\
\hline 17 A $23 \mathrm{Pt}$ & 0,064 & 27,26 & 0,658 & 0,000 & 3,695 & 9,76 \\
\hline $10 \mathrm{~A} 23 \mathrm{Pt}$ & $-0,209$ & 34,86 & 0,671 & 0,005 & 2,997 & 8,97 \\
\hline 5 A $23 \mathrm{Pt}$ & $-1,003$ & 57,09 & 0,888 & 0,057 & 3,307 & 9,36 \\
\hline
\end{tabular}

${ }^{(1)}$ Número de amostras simples; ${ }^{(2)}$ Número de pontos amostrais; ${ }^{(3)}$ Coeficiente de determinação; ${ }^{(4)}$ Raiz quadrada do erro médio; ${ }^{(5)}$ Erro médio percentual.

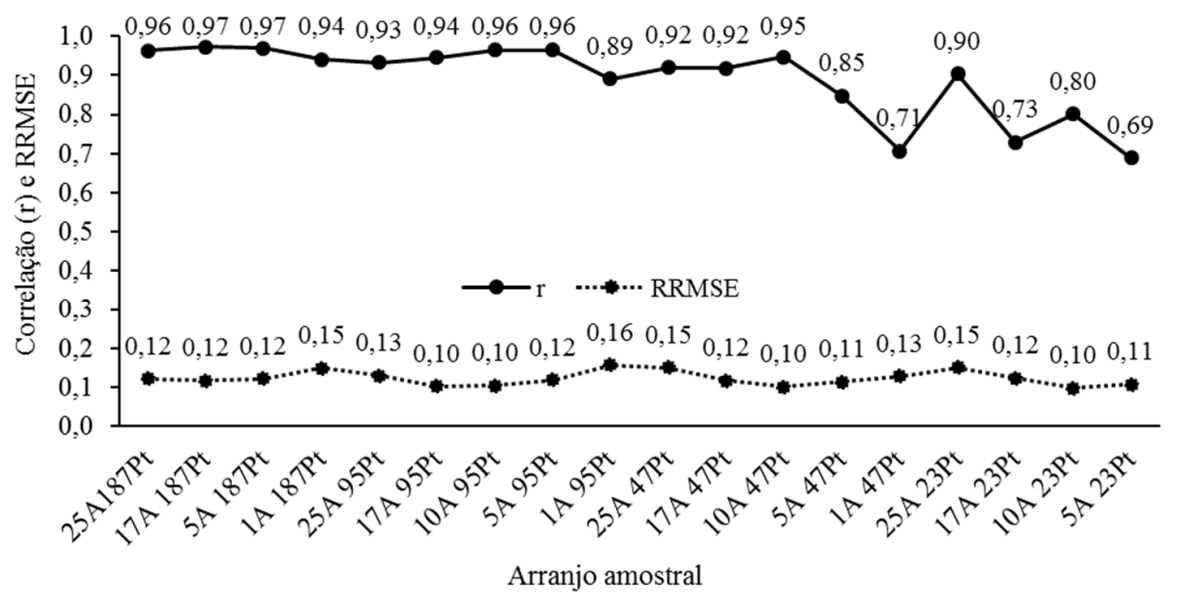

Figura 5. Correlação (r) e valores de RRMSE do mapa de variabilidade com melhor ajuste do variograma e validação cruzada para o teor de MO (10A 187Pt) comparado com os mapas dos restantes arranjos amostrais.

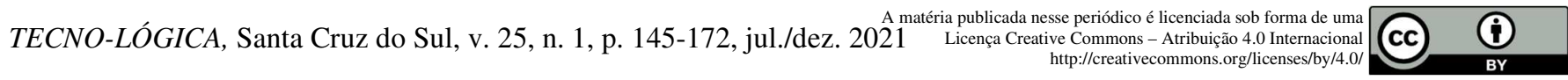




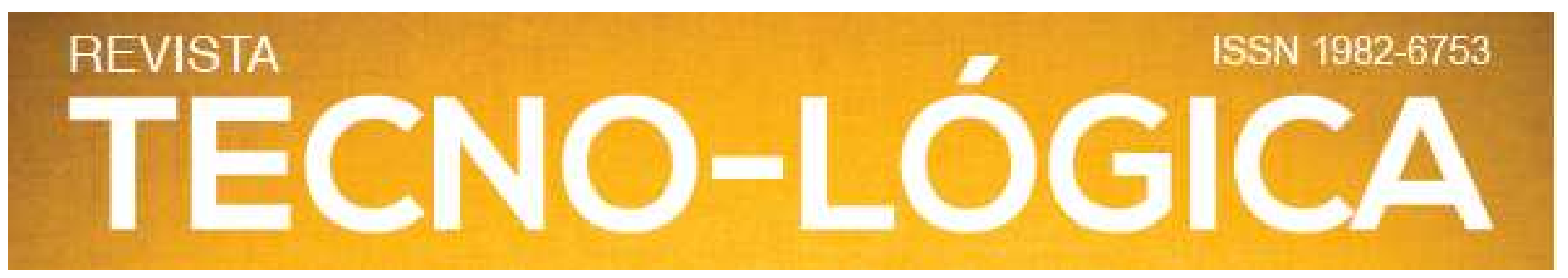

Para os teores de argila, observa-se que houve ajustes semelhantes em todas as malhas amostrais, com exceção do arranjo com 23 pontos. O modelo gaussiano foi o que melhor explicou a variabilidade horizontal da argila no solo, com forte dependência espacial (Tabela 12).

Na Tabela 13 estão apresentados os parâmetros da validação cruzada para o teor de argila do solo. Ficou evidente a redução da qualidade das estimativas com o uso de poucas amostras para a krigagem. No entanto, mesmo com apenas 23 pontos, houve melhor ajuste do modelo teórico para estimar a variabilidade espacial da argila, quando comparado aos atributos químicos do solo, mesmo quando, para esses, se utilizou as malhas mais densas. Segundo a classificação utilizada por Nanni et al. [4], o coeficiente de determinação da validação cruzada foi considerado bom $\left(0,5 \leq \mathrm{R}^{2} \leq 0,75\right)$ para o arranjo contendo 23 pontos e excelente $\left(R^{2}>0,75\right)$ para os arranjos com mais pontos.

Tabela 12. Parâmetros dos modelos teóricos dos semivariogramas ajustados para o teor de argila do solo nos diferentes arranjos amostrais utilizados

\begin{tabular}{|c|c|c|c|c|c|c|c|c|}
\hline Arranjo & Pontos excluídos & Modelo & Alcance (m) & $\mathrm{C}_{0}+\mathrm{C}^{(3)}$ & $\mathrm{C}_{0}{ }^{(4)}$ & IDE $^{(5)}$ & $\mathrm{SQR}^{(6)}$ & $\mathbf{R}^{2(7)}$ \\
\hline $25 \mathrm{~A}^{(1)} 187 \mathrm{Pt}^{(2)}$ & 0 & $\mathrm{GAU}^{(8)}$ & 431 & 6630 & 260 & 96,1 & 139711 & 0,99 \\
\hline 25 A $95 \mathrm{Pt}$ & 0 & GAU & 465 & 6710 & 300 & 95,5 & 83270 & 0,99 \\
\hline $25 \mathrm{~A} 47 \mathrm{Pt}$ & 0 & GAU & 406 & 5549 & 380 & 93,1 & 29572 & 0,99 \\
\hline $25 \mathrm{~A} 23 \mathrm{Pt}$ & 0 & GAU & 513 & 6321 & 1090 & 82,7 & 107130 & 0,99 \\
\hline
\end{tabular}

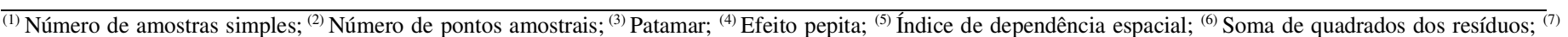
Coeficiente de determinação; ${ }^{(8)}$ Gaussiano.

Tabela 13. Validação cruzada dos modelos teóricos de semivariância ajustados para o teor de argila do solo nos diferentes arranjos amostrais utilizados

\begin{tabular}{|c|c|c|c|c|c|c|}
\hline Arranjo & Coeficiente de regressão & Intercepto $(\mathbf{Y})$ & Erro padrão (SE) & $\mathbf{R}^{2(3)}$ & RQEM $^{(4)}$ & $\mathbf{E M P}^{(5)}$ \\
\hline $25 \mathrm{~A}^{(1)} 187 \mathrm{Pt}^{(2)}$ & 1,037 & $-20,74$ & 0,023 & 0,92 & 18,10 & 2,56 \\
\hline 25 A $95 \mathrm{Pt}$ & 1,028 & $-15,62$ & 0,035 & 0,90 & 18,75 & 2,79 \\
\hline $25 \mathrm{~A} 47 \mathrm{Pt}$ & 0,993 & 3,69 & 0,063 & 0,84 & 23,44 & 3,42 \\
\hline 25 A $23 \mathrm{Pt}$ & 1,129 & $-68,74$ & 0,155 & 0,72 & 32,11 & 4,89 \\
\hline
\end{tabular}

${ }^{(1)}$ Número de amostras simples; ${ }^{(2)}$ Número de pontos amostrais; ${ }^{(3)}$ Coeficiente de determinação; ${ }^{(4)}$ Raiz quadrada do erro médio; ${ }^{(5)}$ Erro médio percentual.

Para os teores de argila do solo foram observadas correlações muito fortes entre os mapas dos diferentes arranjos amostrais, além de baixos valores de RRMSE (Figura 6). Como a argila é pouco influenciada pelo manejo adotado no talhão, a variação horizontal existente é resultado somente de processos pedogenéticos que atuaram sobre os diferentes materiais de origem, conhecidos como macro variações. Normalmente, as macrovariações se correlacionam espacialmente e apresentam altos valores de alcance da dependência espacial [26].

TECNO-LÓGICA, Santa Cruz do Sul, v. 25, n. 1, p. 145-172, jul./dez. 2021 A matéria publicada nesse periódico é licenciada sob forma de uma 


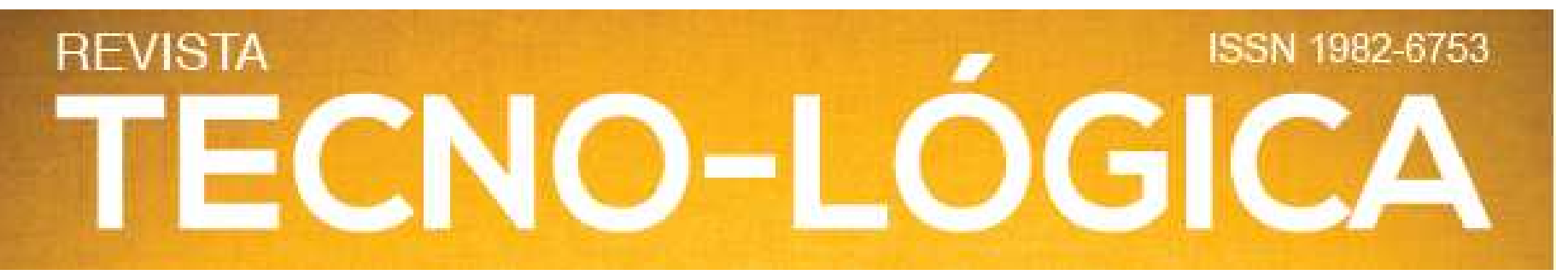

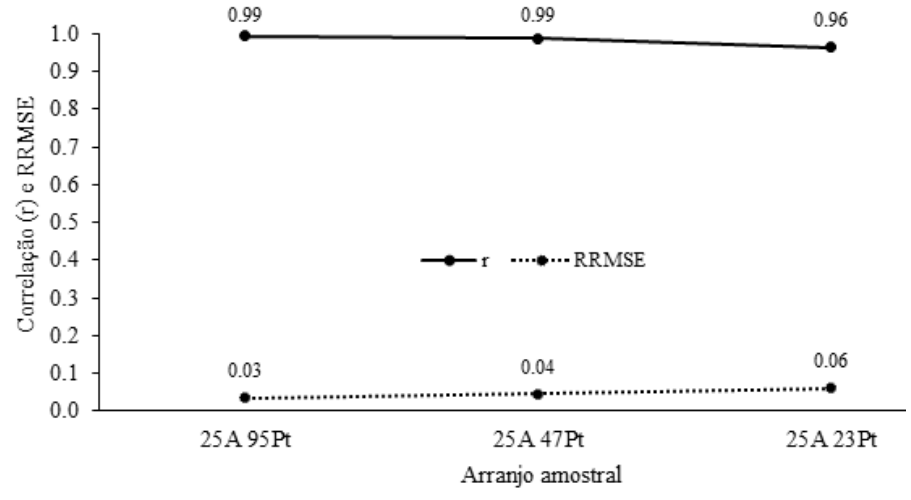

Figura 6. Correlação (r) e valores de RRMSE do mapa de variabilidade com melhor ajuste do variograma e validação cruzada para a argila (25A 187Pt) comparado com os mapas dos restantes arranjos amostrais.

Por outro lado, para os atributos químicos do solo, K, V\% e principalmente o $\mathrm{P}$, mesmo apresentando dependência espacial moderada a forte e bons ajustes de modelos teóricos, não foram observados bons valores de $\mathrm{R}^{2}$ da validação cruzada, indicando que existe uma grande dispersão da nuvem de pontos em torno da reta ideal. A baixa precisão das estimativas pode ocorrer devido às variações extrínsecas causadas pela aplicação de fertilizantes, práticas de cultivo e erosões ocorridas [1].

Para este estudo, foi utilizada uma malha amostral densa, principalmente quando comparada às malhas que se utilizam comercialmente no Brasil, e não foram obtidos bons parâmetros da validação cruzada para os atributos químicos estudados. Esse resultado pode sugerir que, mesmo com malhas amostrais mais intensas, não há melhoria na precisão dos valores estimados pela krigagem. As práticas de correção e de adubação do solo ao longo dos anos de cultivo podem ter ocasionado mudanças intensas na variabilidade horizontal desses atributos, que inviabilizam o ajuste de modelos teóricos que explicam a variação dos mesmos.

Em contrapartida, a variabilidade espacial dos teores argila do solo foi estimada com boa precisão, mesmo com um conjunto pequeno de dados. Esse resultado contradiz aos observados por Kerry e Oliver [16] e Souza et al. [22], por meio dos quais afirmaram a ocorrência de diminuição da precisão da estimativa do variograma quando se usa menos que 100 pontos amostrais. Dessa forma, supõe-se que, se a distância entre os pontos for suficiente para se captar a dependência espacial do atributo estudado, e este não tenha sofrido intensa influência do manejo na sua variabilidade, um pequeno conjunto de dados amostrais permitem modelar com precisão a sua variabilidade espacial, concordando com os resultados obtidos por Ragagnin et al. [3].

Levando-se em consideração os ajustes dos modelos dos variogramas, a validação cruzada, a correlação entre os mapas, e os valores de RRMSE, observa-se que a utilização de apenas uma amostra simples por ponto amostral prejudica a modelagem da variabilidade horizontal dos atributos químicos do solo, principalmente o P e K. O resultado discorda com os de Siqueira et al. [6], no entanto, esses autores compararam as correlações entre os mapas de argila, susceptibilidade magnética e saturação

TECNO-LÓGICA, Santa Cruz do Sul, v. 25, n. 1, p. 145-172, jul./dez. $2021 \quad \begin{gathered}\text { A matéria publicada nesse periódico é licenciada sob forma de uma } \\ \text { Licença Creative Commons - Atribuição } 4.0 \text { Internacional }\end{gathered}$ 


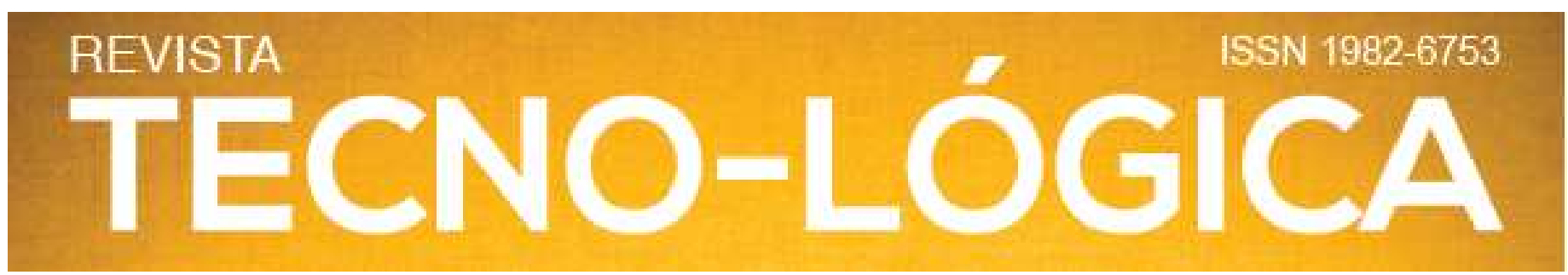

por bases com amostras formadas por uma e três amostras simples somente.

Pelos resultados apresentados, observa-se maior influência e importância do número de amostras simples para os nutrientes potássio e fósforo. Entretanto, para todos os atributos avaliados, houve perda de qualidade dos semivariogramas e validação cruzada, além de diminuição da correlação entre os mapas de variabilidade quando se utiliza apenas uma amostra simples por ponto. Ao contrário do esperado, não foi observado aumento de qualidade quando se utilizou mais de 17 amostras simples por ponto. A possível homogeneização ocasionada pela utilização de subamostragem não foi observada, pois não houve diminuição evidente do IDE dos modelos de semivariogramas ajustados quando foi utilizado mais de uma amostra simples por ponto.

Em função da influência do número de amostras simples nos parâmetros da qualidade da interpolação, foram ajustadas equações de regressão para os parâmetros de avaliação da qualidade da interpolação, sendo verificado o efeito significativo do número de amostras utilizadas $(\mathrm{p}<0,01)$ em todos os parâmetros testados (Figura 7).
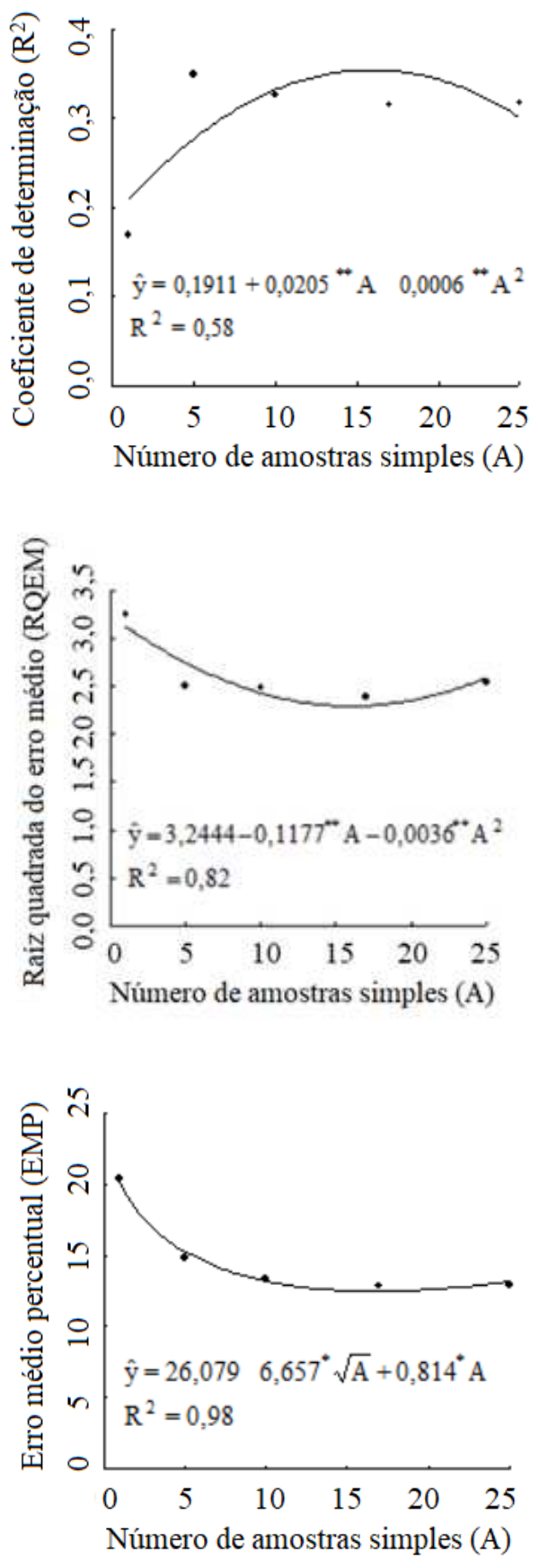

Figura 7. Gráficos de regressão dos parâmetros de qualidade da interpolação

TECNO-LÓGICA, Santa Cruz do Sul, v. 25, n. 1, p. 145-172, jul./dez. $2021 \quad \begin{gathered}\text { A matéria publicada nesse periódico é licenciada sob forma de uma } \\ \text { Licença Creative Commons - Atribuição } 4.0 \text { Internacional }\end{gathered}$ http://creativecommons.org/licenses/by/4.0/ 


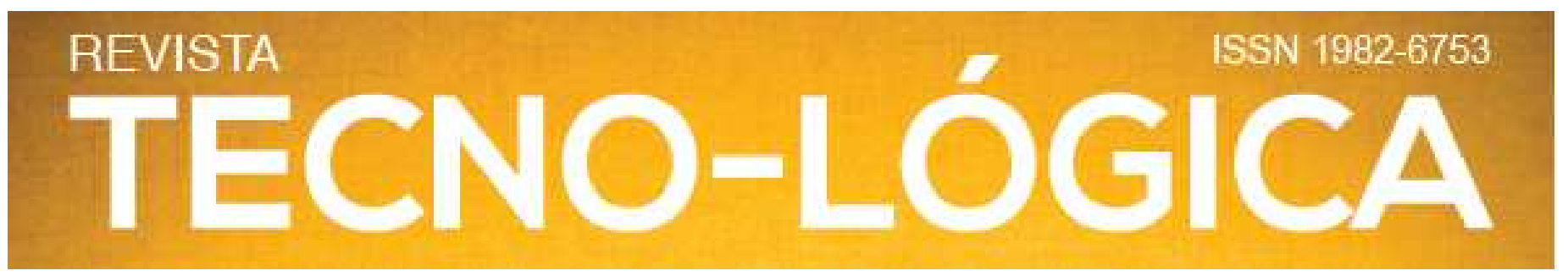

O ponto de máximo coeficiente de determinação da validação cruzada $(0,354)$ foi estimado com 15,9 amostras simples e os mínimos da RQEM e do EMP (2,29 e 12,47, respectivamente) foram estimados com 16,0 e 16,7 amostras simples por ponto, respectivamente. Calculando-se o número de amostras necessárias para atingir 90\% dos valores máximo e mínimos, obtêm-se 8,6; 7,7

e 7,7 amostras simples por ponto para o $R^{2}$ da validação cruzada, a RQEM e o EMP, respectivamente. Dessa forma, utilizando-se 8 amostras simples por ponto, obteremos um $\mathrm{R}^{2}$ de validação cruzada de 0,314, uma RQEM de 2,53 e um EMP de 13,76\%, todos com valores atingindo acima dos $89,9 \%$ do melhor valor calculado, com redução de 8 de amostras simples (50\%) por ponto amostral em média, já que os pontos de máxima e mínimas dos três parâmetros testados ficaram em torno de 16 amostras simples por ponto.

\section{Conclusões}

No presente artigo, foi detectada a dependência espacial para todos os atributos do solo avaliados.

Houve diferença entre a qualidade dos variogramas, da validação cruzada e dos mapas de variabilidade espacial dos atributos gerados com diferentes números de amostras simples, sendo necessárias oito amostras simples por ponto para uma boa qualidade da estimativa dos atributos do solo.
A utilização de apenas uma amostra simples por ponto amostral, prejudica a modelagem da variabilidade horizontal dos atributos do solo, principalmente do fósforo e potássio.

São necessários 1 ponto amostral para cada 0,54 ha (187 pontos) para estimar o fósforo, o potássio e a saturação por bases, 1 ponto para cada 1,06 ha (95 pontos) para estimar o $\mathrm{pH}$ e a matéria orgânica e, 1 ponto para cada 4,39 ha (23 pontos) para estimar a argila do solo.

Os atributos do solo que sofrem menor influência de fatores extrínsecos são os que apresentam as melhores estimativas em locais não amostrados.

\section{Agradecimentos}

Ao Sr. Florino Wielemaker, pela colaboração e cessão da área estudada; ao CNPQ, pela concessão da bolsa de estudos; e a Danilo Gomes Fortes, Eduardo Messias de Andrade Almeida, Eliéser de Almeida e Matheus Andrade Martinez, pelo auxílio na condução dos trabalhos de campo.

\section{DEPENDENCE AND SPATIAL CORRELATION OF SOIL ATTRIBUTES AS FUNCTION OF SAMPLE \\ ARRANGEMENT}

ABSTRACT: National agriculture has undergone intense changes, both in the way of managing the properties and in the 


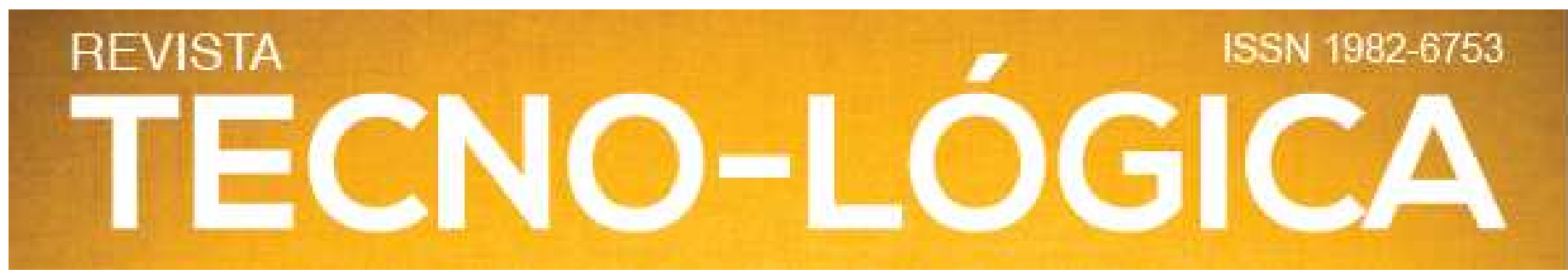

technological level employed in them. Among the technologies adopted, emphasis is placed on precision agriculture, mainly used for the application of fertilizers at various rates, using the information from the analysis of soil samples, collected in georeferenced continuous grids. The present study was conducted in a plot of Fazenda Planalto, located in the municipality of Maracaju - MS, with the objective of understanding the different sampling arrangements adopted for soil collection and its influence on the geostatistical analysis of phosphorus, potassium, base saturation, $\mathrm{pH}$, organic matter and clay content, as well as the reliability of the prediction maps elaborated from the geostatistical analysis. Different numbers of soil simple samples were used to form the soil composite sample (1, 5, 10, 17 and 25) combined with a thinning of the sample points to form different grid resolutions (187, 95, 47 and 23 points). It was concluded that eight simple samples are necessary for a smaller error of prediction of values in non-sampled locations. To estimate spatial dependence, sample grids with: 187 points for potassium phosphorus and base saturation, 95 points for $\mathrm{pH}$ and organic matter and 23 points for clay content are needed. The best estimates in unsampled locations are obtained for the clay.

Keywords: Precision agriculture. Sampling. Sample grid. Spatial variability.

TECNO-LÓGICA, Santa Cruz do Sul, v. 25, n. 1, p. 145-172, jul./dez. 2021 A matéria publicada nesse periódico é licenciada sob forma de uma

TECNO-LOGICA, Santa Cruz do Sul, v. 25, n. 1, p. 145-172, jul./dez. 2021 Licença Creative Commons - Atribuição 4.0 Internacional

\section{Referências}

[1] CAMBARDELlA, C. A.; MOORMAN, T. B.; NOVAK, J. M.; PARKIN, T. B.; KARLEN, D. L.; TURCO, R. F.; KONOPKA, A. E. Field-scale variability of soil properties in central Iowa soils. Soil Science Society America Journal, Madison, Vol. 58, n. 4, p. 1501-1511, 1994

[2] CAVAlCANTE, E. G. S.; ALVES, M. C.; PEREIRA, G. T.; SOUZA, Z. M. (2007). Variabilidade espacial de MO, P, K, e CTC do solo sob diferentes usos e manejos. Ciência Rural, Santa Maria, Vol. 37, n. 2, p. 394-400, 2007.

[3] RAGAGNiN, A. V.; SENA JUNIOR, D. G.; SILVEIRA NETO, A. N. Recomendação de calagem a taxa variada sob diferentes intensidades de amostragem. Revista Brasileira de Engenharia Agrícola e Ambiental, Campina Grande, Vol. 14, n. 6, p. 600-607, 2010

[4] NANNI, M. R.; POVH, F. P.; DEMATTÊ, J. A. M.; OLIVEIRA, R. B. DE; CÉZAR, E. Optimum size in grid soil sampling for variable rate application in sitespecific management. Scientia Agricola, Piracicaba, Vol. 68, n. 3, p. 386-392, 2011.

[5] CAON, D.; GENÚ, A. M. Mapeamento de atributos químicos em diferentes densidades amostrais e influência na adubação e calagem. Revista Brasileira de Engenharia Agrícola e Ambiental, Campina Grande, Vol. 17, n. 6, p. 629-639, 2013.

[6] SIQUEIRA, D. S.; MARQUES JUNIOR, J.; PEREIRA, G. T.; BARBOSA, R. S.; TEIXEIRA, D. B.; PELUCO, R. G. Sampling density and proportion for the characterization of the variability of Oxisol atributes on diferente materials. Geoderma, Elsevier, Vol. 232-234, p. 172-182, 2014.

[7] GUARÇONI, A, M.; ALVAREZ, V. H.; NOVAIS, R. F.; CANTARUTTI, R. B.; LEITE, H. G.; FREIRE, F. M. Definição da dimensão do indivíduo solo e determinação do número de amostras simples necessário à sua representação. Revista Brasileira de Ciência do Solo, Viçosa, Vol. 30, n. 6, p. 943-954, 2006. 


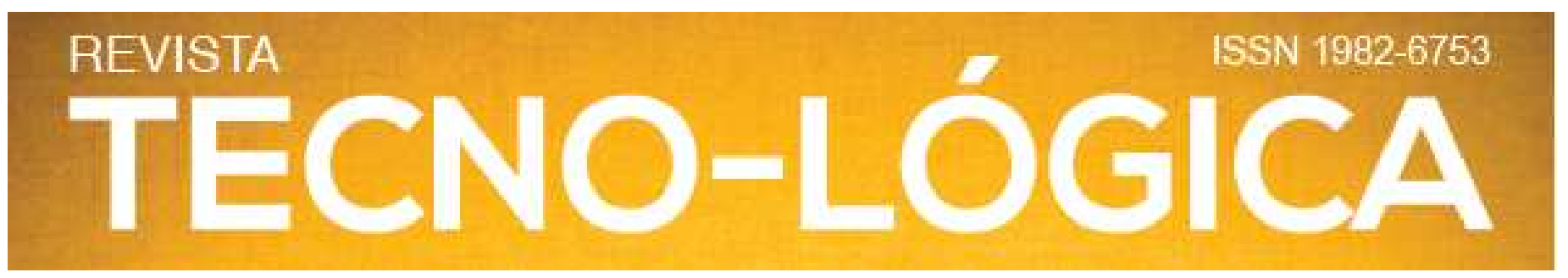

[8] SCHLINDWEIN, J. A.; ANGHINONI, L. Variabilidade horizontal de atributos de fertilidade e amostragem do solo no sistema de plantio direto. Revista Brasileira de Ciência do Solo, Viçosa, Vol. 24, n. 1, p. 85-91, 2000.

[9] ALVARES, C. A.; STAPE, J. L.; SENTELHAS, P. C.; GONÇALVES, J. L. M.; SPAROVEK, G. Köppen's climate classification map for Brazil. Meteorologische Zeitschrift, Vol. 22, n. 6, p. 711-728, 2013.

[10] CLAESSEN, M. E. C., (Org.). Manual de Métodos de análises de solo. (2.ed.). Rio de Janeiro, 1997. 212p.

[11] LIBARDI, P. L.; MANFRON, P. A.; MORAES, S. O.; TUON, R. L. Variabilidade da umidade gravimétrica de um solo hidromórfico. Revista Brasileira de Ciência do Solo, Viçosa, Vol. 20, n. 1, p. 01-12, 1996.

[12] ROBERTSON, G. P. GS+: Geostatistics for the environmental sciences - GS + User’s Guide. Plainwell, Gamma Desing Software, 2008. 152p.

[13] ZIMBACK, C. R. L. Análise espacial de atributos químicos de solos para fins de mapeamento da fertilidade do solo. Tese de Livre Docência. Universidade Estadual Paulista, Botucatu, 2001. 114p.

[14] KRAVCHENKO, A. N. Influence of spatial structure on accuracy of interpolation methods. Soil Science Society of America Journal, Madison, Vol. 67, n. 5 , p. $1564-1571,2003$.

[15] KERRY, R.; OLIVER, M. A. Comparing sampling needs for variograms of soil properties computed by the method of moments and residual maximum likelihood. Geoderma, Elsevier, Vol. 140, p. 383-396, 2007.

[16] KERRY, R.; OLIVER, M. A. Determining nugget: sill ratios of standardized variograms from aerial photographs to krige sparse soil data. Precision Agriculture, Vol. 9, p. 33-56, 2008.

[17] MUKAKA, M. M. A guide to appropriate use of correlation coefficient in medical research. Malawi Medical Journal, Vol. 24, n. 3, p. 69-71, 2012.
[18] LI, J.; HEAP, A. D. A. Review of Spatial Interpolation Methods for Environmental Scientists. Geoscience Australia, Record 2008/23, 2008. 137 p.

[19] FERREIRA, D. F. Sisvar: a Guide for its Bootstrap procedures in multiple comparisons. Ciência e Agrotecnologia.Lavras [online]. Vol. 38, n. 2, p. 109-112, 2014.

[20] WILDING, L. P.; DREES, L. R. Spatial variability and pedology. In L. P. Wilding, \& L. R. Drees (Eds). Pedogenesis and soil taxonomy: concepts and interactions, Elsevier, p. 83-116. 1983.

[21] EMBRAPA. Empresa Brasileira de Pesquisa Agropecuária. Tecnologias de produção de soja - região central do Brasil - 2012 e 2013. Londrina, Embrapa Soja, 2011. $261 \mathrm{p}$.

[22] SOUZA, Z. M.; SOUZA JUMIOR, G. S.; J. M.; PEREIRA, G. T. Número de amostras na análise geoestatística e na krigagem de mapas de atributos do solo. Ciência Rural, Santa Maria, Vol. 44, n. 2, p. 261-268, 2014.

[23] CORÁ, J. E.; ARAUJO, A. V.; FERREIRA, G. T.; BERALDO, J. M. G. Variabilidade espacial de atributos do solo para adoção do sistema de agricultura de precisão na cana-de-açúcar. Revista Brasileira de Ciência do Solo, Viçosa, Vol. 28, n. 6, p. 1013-1021, 2004.

[24] ZANÃO JUNIOR, L. A.; LANA, R. M. Q.; GUIMARÃES, E. C. Variabilidade espacial do $\mathrm{pH}$, teores de matéria orgânica e micronutrientes em profundidade em um Latossolo Vermelho sob semeadura direta. Ciência Rural, Vol. 37, p. 1000-1007, 2007.

[25] SILVA, S. A.; LIMA, J. S. S.; SOUZA, G. S.; OLIVEIRA, R. B.; SILVA, A.

F. Variabilidade espacial do fósforo e das frações granulométricas de um Latossolo Vermelho Amarelo. Revista Ciência Agronômica, Vol. 41, n. 1, p. 1-8, 2010.

[26] MELLO, G.; BUENO, C. R. P.; PEREIRA, G. T. Variabilidade espacial das propriedades físicas e químicas do solo em áreas intensamente cultivadas. Revista

TECNO-LÓGICA, Santa Cruz do Sul, v. 25, n. 1, p. 145-172, jul./dez. $2021 \stackrel{\text { A matéria publicada nesse periódico é licenciada sob forma de uma }}{\text { Licença Creative Commons - Atribuição } 4.0 \text { Internacional }}$ http://creativecommons.org/licenses/by/4.0/ 


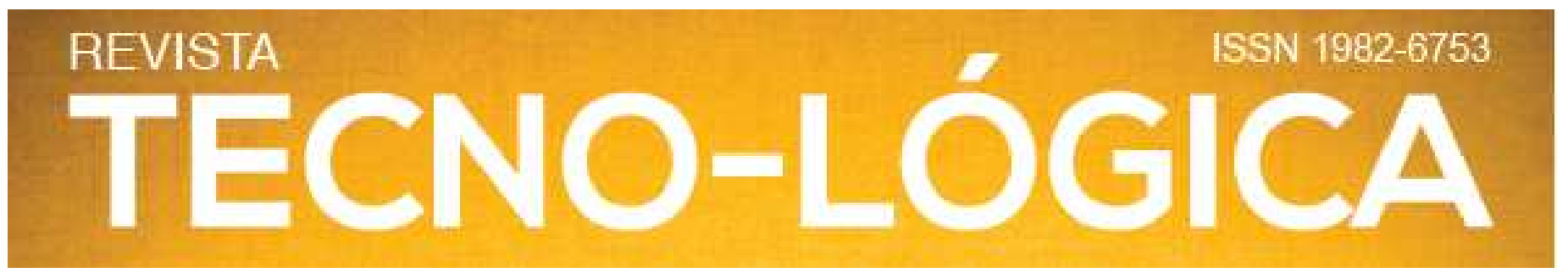

Brasileira de Engenharia Agrícola e Ambiental, Campina Grande, Vol. 10, n. 2, p.

294-305, 2006. 